\title{
Histoire politique du Nagriamel à Santo (Vanuatu)
}

\section{Marc Kurt Tabani}

\section{(2) OpenEdition}

\section{Journals}

\section{Édition électronique}

URL : http://journals.openedition.org/jso/1584

DOI : $10.4000 /$ jso.1584

ISSN : $1760-7256$

\section{Éditeur}

Société des océanistes

\section{Édition imprimée}

Date de publication : 1 décembre 2001

Pagination : 151-176

ISSN : 0300-953x

\section{Référence électronique}

Marc Kurt Tabani, « Histoire politique du Nagriamel à Santo (Vanuatu)», Journal de la Société des

Océanistes [En ligne], 113| Année 2001-2, mis en ligne le 27 mai 2008, consulté le 01 mai 2019. URL : http://journals.openedition.org/jso/1584 ; DOI : 10.4000/jso.1584 


\section{Histoire politique du Nagriamel à Santo (Vanuatu)}

par

Marc Kurt TABANI*

\section{RÉSUMÉ}

Cet article retrace l'histoire politique Nagriamel dans l'île de Santo à Vanuatu et vise à expliquer, à travers l'examen d'éléments biographiques relatifs à son leader charismatique Jimmy Stevens, les dérives traditionalistes de ce mouvement. Ce dernier fut le principal artisan de l'unification du "peuple du dark bush" (i.e. les populations isolées de l'intérieur de l'île) sous l'égide d'une coutume (kastom) assemblée de toutes pièces. L'examen du parcours de ce personnage hors du commun qui du statut de boy au service des colons devint un " roi des îles » et " prince » autoproclamé de la "coutume de Santo » et de l'instrumentalisation qu'il fit des revendications nativistes de ses partisans autochtones, sera également l'occasion de revenir sur cette période troublée de l'accession à l'indépendance de la République de Vanuatu.

Mots clés : Nagriamel ; Jimmy Stevens ; histoire de Santo ; anthropologie politique, Kastom ; traditionalisme, invention des traditions, Vanuatu.

Le mouvement politique qui s'est développé après-guerre dans l'île de Santo, à Vanuatu, sous le nom de Nagriamel, se prête particulièrement bien à illustrer comment l'aspect libérateur d'un mouvement indigéniste peut se changer en la réinvention traditionaliste d'une organisation sociale pré-existante. Il fut précédé à Santo par

\section{ABSTRACT}

This article deals with the history of the Nagriamel, a political movement in the Santo island (Vanuatu) and tries to explain its traditionalist drifts by the bias of biographical aspects concerning its charismatic leader Jimmy Stevens. Stevens was the originator of the unification of the "dark bush people " (the enclaved communities of the inland of Santo) by the way of an assemblage of ill assorted customs. The illustration of the personnal history of this incredible personnage, who started his carrer as a boy, a servant of his colonial masters and became an "island king », an autoproclamated " prince » of "Santo's custom », and the examination of the way he had instrumentalised the claims of his native faction, will also be the occasion to throw some lights on the troubled times of independence gaining in Vanuatu.

Key words : Nagriamel ; Jimmy Stevens ; History of Santo, Political Anthropology; Kastom ; Traditionalism ; Invention of Tradition ; Vanuatu.

des manifestations nativistes, syncrétiques et millénaristes, d'une moindre notoriété (mouvements Avu Avu, Rongofuro, Naked Cult, cf. Raff, 1928 ; Miller, 1948 ; Guiart, 1958), dont il s'est opportunément et sélectivement inspiré. Rappelons que parmi les types de revendications identitaires philo-coutumières qu'il distingue en

* Docteur en Ethnologie de l'Université Montpellier III ; responsable des collections d'ethnologie à la Bibliothèque nationale de France. 
Mélanésie, R. M. Keesing fait explicitement référence au Nagriamel pour illustrer la combinaison d'un discours sur la tradition (kastom) et de choix politiques, au travers de laquelle se cristallise une force d'engagement susceptible de déclencher des crises séparatistes dans le contexte des édifications nationales en Mélanésie :

« La rhétorique de la coutume [kastom] est invoquée en référence à une région ou à une île précise à l'intérieur de l'État post-colonial. Celle-ci peut prendre la forme d'une compétition pour les ressources ou le pouvoir politique de l'État, d'un séparatisme régional, ou même de revendications sécessionnistes » (Keesing, 1989 : 21) [traduction de l'auteur].

Les revendications traditionalistes régionalistes sont à situer à un niveau d'engagement intermédiaire, entre une revendication kastom fondée sur des bases syncrétiques traditionnellement adaptées (comme le mouvement millénariste John Frum à Tanna, $c f$. Tabani, 1999) et un néotraditionalisme d'État idéologiquement tourné vers le nationalisme ( $c f$. mon analyse de la coutume d'État à Vanuatu, Tabani, 2000 et 2001). À Santo, le rôle précoce de la conscience d'un déclin des anciennes traditions pour des cultures encore vécues s'est significativement intensifié pendant l'épisode de la Seconde Guerre mondiale et l'arrivée successive sur l'île de centaines de milliers d'hommes de troupes américains, avec toute leur puissance technologique, et la présence singulière des soldats noirs " égaux " des Blancs ${ }^{1}$. Ce n'est qu'à l'horizon d'une période post-coloniale qu'un mouvement comme le Nagriamel s'engagea délibérément dans une revendication traditionaliste qui, à la différence des précédents cultes, ne permit plus de réduire des contradictions culturelles entre le passé et le présent ; au contraire, il les accentuait. Les vélléités séparatistes du Nagriamel semblent correspondre à ce point de basculement où les incompatibilités entre le mode de vie ancestral propre à des enclaves culturelles et leur inscription moderne dans un contexte global, cessent d'être médiatisées par des voies principalement rituelles.

L'examen de la revendication kastom exprimée par le Nagriamel demande à ce que l'on ne perde de vue ni la nature des mouvements dont il s'est inspiré, ni les particularités de son histoire politique dans un contexte colonial. De même, que ce soit face aux défis de la modernité et aux enjeux de l'adaptation culturelle dans la diversité de ses recompositions sociologiques, ou encore, devant le poids des influences extérieures dans ses orientations idéologiques, les spécificités de la stratégie du Nagriamel demeureraient inintelligibles si l'on ne rappelait pas le rôle central joué par son leader historique : Jimmy Moses Tubo Pantuntun Moli Stevens, plus généralement connu sous le nom de Jimmy Stevens. L'analyse de la revendication kastom du Nagriamel est à mettre en parallèle avec les éléments autobiographiques que Stevens mit au profit de la notoriété de ce mouvement pour le plus grand bénéfice personnel de son pouvoir autocratique et de son statut de leader prophétique. Cet aspect individuel et charismatique dans les revendications concernant la kastom, me fournira l'occasion de souligner, comment, sur un plan local ou national, traditionaliste ou néo-traditionaliste, historique ou actuel, celle-ci pose invariablement la question de l'action des « inventeurs » de traditions, c'est-à-dire du rapport de sujets autonomes à leur «tradition », et des personnifications charismatiques -extra-traditionnelles- du discours identitaire sur la kastom.

Enfin, en dernier lieu, il me faudra examiner le Nagriamel sous l'angle d'un traditionalisme qui, malgré ses transformations et ses velléités sécessionnistes, n'a jamais véritablement atteint une dimension nationaliste. Précurseur du « modernisme-coutumier » ( $c f$. Babadzan, 1988), voire dans son cas d'un "hyper-libéralisme traditionaliste » comme nous l'examinerons plus loin, le Nagriamel était accessoirement nativiste et marginalement syncrétique. Toutefois, bien qu'il soit traditionaliste et sélectif, il n'opposa cependant pas de refus dogmatique au changement, conservant ainsi une part de flexibilité traditionnelle. En ayant voulu symboliquement se présenter comme le continuateur patenté de cultes syncrétiques qui l'ont précédé, le Nagriamel s'est mis à gérer un héritage nativiste (anti-Blancs), revivaliste (contre-colonial puis anti-national) et millénariste (cargoïste).

Néanmoins, les particularités syncrétiques récupérées par le traditionalisme du Nagriamel n'offraient qu'un bien faible contrepoids aux influences et aux manipulations extérieures dont a fait l'objet ce mouvement. Le Nagriamel ne s'est véritablement détaché de ses affiliations coloniales francophiles que dans les moments où il se risquait dans des opérations néo-coloniales hyper-libérales et radicalement anti-coutu-

1. Il ressort d'une étude de Lindstrom sur la présence massive des Américains en Mélanésie pendant la bataille de la mer de Corail, que les revendications philo-coutumières ont eu tendance, à cette période, à s'effacer au profit d'un apprentissage de la modernité et d'une découverte des pratiques égalitaires des Américains, et au détriment de l'autorité coloniale ( $c f$. Lindstrom, 1996 : 17). 
mières. C'est toute la complexité socio-politique de ce traditionalisme et de son caractère utopique prononcé que je chercherai à clarifier.

\section{L'Act of Dark Bush et la fondation de Vanafo}

L'histoire politique de la revendication kastom connut, à Santo, de singuliers développements. La rébellion qui intervint, lors la proclamation de l'indépendance de Vanuatu, dans la plus grande et la plus riche des îles de cet archipel, inscrivit les péripéties du Nagriamel en bas de page de l'histoire de la décolonisation. La nouvelle fit le tour des grandes capitales; une lutte insolite se déroulait dans ces îles du bout du monde. Beasant (1984: 17), ancien conseiller de Walter Lini, rapporte la teneur de ce cliché :

« L'ex-conducteur de bulldozer devenu chef d'un genre bien particulier, avec ses 'vingt-cinq femmes', et conduisant une 'armée de l'âge-de-pierre', armée 'd'arcs et de flèches', engagée dans une rébellion contre les forces matérielles et les conceptions d'un insensible monde extérieur » [traduction de l'auteur].

Loin de telles évocations fantasmagoriques, on trouve à l'origine du lancement du Nagriamel un vaste processus de dépossession foncière des terres indigènes. Menée sur l'initiative des colons européens de Santo, cette entreprise prit de l'ampleur après-guerre, principalement du fait des routes carrossables qui avaient été ouvertes par les Américains en direction de l'intérieur de l'île, vers des zones évacuées de leurs populations de par leur proximité avec les camps militaires. Les terres les plus convoitées étaient celles se trouvant en amont de la rivière Sarakata, dans un domaine foncier connu sous le nom de «Luganville Estate ». La propriété de ce territoire fut attribuée, pour quatre-vingt-dix-neuf ans, à la Société Française des NouvellesHébrides (SFNH), par deux jugements du tribunal condominial en 1951 et 1959.

L'initiative d'une contestation indigène fut prise, durant les années soixante, par Paul Buluk, "chef coutumier» de l'intérieur de Santo, qui réclamait des droits fonciers sur une partie des terres de la SFNH, et dont il entendait prouver qu'elles avaient été occupées par ses propres aïeuls. De là date sa rencontre avec Jimmy Stevens qui fut, quelques années auparavant, conducteur de bulldozer. Il avait, à ce titre, procédé lui-même, sur ordre de la compagnie qui l'embauchait, à la destruction des vestiges du village ancestral de Buluk, y compris les tombes des parents de ce dernier. Stevens avait été approché par les man-bush pour le témoignage qu'il pouvait rapporter sur sa participation à cette action et pour ses éventuelles capacités à leur livrer des armes, le trafic de marchandises étant alors sa principale activité.

Les sollicitations de Buluk auprès de Stevens dépassèrent rapidement la demande d'un simple soutien. Elles se transformèrent en une proposition de prendre la tête d'un mouvement indigène de revendication foncière ${ }^{2}$. La date exacte de la fondation du Nagriamel demeure mal connue. L'officialisation du mouvement remonte à l'année 1965. Dans l'arrière salle d'un bar de Luganville, fut proclamé l' " Act of Dark Bush », pour énoncer le principe de la restitution des terres indigènes annexées et contester toute extension des plantations de colons français. La création du Nagriamel consacrait la convergence définitive des intentions idéologiques de Stevens et de l'engagement sur le terrain de Buluk et de ses partisans du bush.

Les quelques centaines de membres du mouvement estimés pour la période qui précède les années soixante-dix adhérèrent au projet de Buluk et Stevens de s'installer à l'intérieur des limites du domaine très convoité de la SFNH. Cette décision multiplia les conflits que Buluk avait ponctuellement déclenchés sous la forme de saccage des clôtures des plantations des colons. Pour de telles opérations il avait déjà écopé six mois de prison en 1964. Pourvu d'une charte fondatrice et de militants regroupés sur la base d'une propriété collective de la tenure foncière, d'une économie d'autosubsistance et d'une organisation sociale perpétuant un mode de vie hérité des temps pré-coloniaux, il manquait au Nagriamel une assise territoriale. Il fut convenu de la nécessité de disposer d'un lieu symbolique de la nouvelle autorité fédératrice, représentée par l'alliance de Buluk et de Stevens, et d'un territoire suffisamment grand pour permettre la fondation d'un nouveau type de communauté. Cette communauté devait se mettre au service de l'émancipation des indigènes, s'élever contre l'aliénation coloniale et préparer les opprimés du dark bush à leur émancipation politique. Cette Nouvelle Jérusalem fut établie dans un coude de la rivière Sarakata et prit le nom de Vanafo.

En plein cœur du domaine foncier de la SFNH, sur les plateaux en arrière de Luganville, dans un

2. Comme chaque fois qu'il était question de kastom, tout commençait par un « don » : « Lorsque je leur demandais de quel pouvoir ils disposaient pour revendiquer la terre [...], ils me répondirent, aucun, sinon leurs coutumes traditionnelles [traditional customs]. Mais ils ne savaient comment ils pouvaient les utiliser au mieux, et comptaient sur moi pour leur donner la solution » (Stevens, in Van Trease, 1987 : 139). 
espace déboisé en amont des chutes de la rivière Sarakata au-delà duquel s'étendent d'épaisses jungles, la communauté de Vanafo représentait à l'origine, quelques cases d'un hameau édifié sur les terres revendiquées par Buluk. Le nom Vanafo, "panier de fruits » dans la langue locale, vint symboliser la richesse des terres destinées à nourrir les hommes du Nagriamel. Ce hameau passera rapidement à la taille d'un village et devint le quartier général de l'administration du Nagriamel. Cette installation fut décisive pour les succès ultérieurs que connut ce mouvement, notamment du point de vue de son expansion au-delà des limites de l'île de Santo. Le nom de Nagriamel fut pareillement conçu pour marquer une transition des petits hameaux isolés traditionnels vers une grande concentration communautaire dotée d'une organisation centralisée. Son étymologie provient des noms de deux plantes aux divers usages rituels ou utilitaires: nangaria (Cordyline fruticosa) et namele (Cycas circinalis). À une question de Beasant sur la kastom de Santo, Stevens lui répondit :

« La feuille de namele est notre tabou, notre loi, notre coutume, la feuille de nagria est notre sérénité, notre corps » (Beasant, 1984: 17) [traduction de l'auteur].

De la stratégie échafaudée par Jimmy Stevens à partir de la fondation d'un " quartier général des populations indigènes ", il se dégagea une nécessité d'élargir la base du mouvement et d'obtenir les moyens de son engagement. Des circonstances favorables à la réussite du mouvement s'ouvrirent à Stevens au cours de sa condamnation, en 1968, à six mois de prison en compagnie de Buluk, suite à une tentative d'extension du village de Vanafo. Stevens mit à profit sa détention pour repenser ses perspectives. Il finit par se convaincre de la nécessité d'une politique d'ouverture du Nagriamel, en associant plusieurs facteurs : 1/ l'abandon résolu de l'anti-colonialisme français, dans la perspective d'un soutien de la France; 2/ L'élargissement géographique du Nagriamel en direction des populations du Nord de l'archipel et un plan favorisant leur émigration à Santo ; 3/ La prise de pouvoir de Jimmy Stevens sur le Nagriamel et la légitimation de son autorité par les man-bush sur une base coutumière. L'imposition de ces trois orientations visait à pérenniser ce mouvement, en ne l'enfermant pas dans la seule revendication foncière des man Santo. Telles furent les lignes de conduite retenues par Stevens pour lancer un développement économique, sur la base de moyens technologiques modernes, au profit de ses sympathisants réinvestis dans leurs droits fonciers.

La communauté de Vanafo connut à ses débuts de difficiles moments. Toutefois, les effectifs du Nagriamel gonflèrent rapidement. Lors d'un meeting tenu à Santo en janvier 1966, Stevens réunit six cents participants, dont un certain nombre venu des autres îles ${ }^{3}$. En ces temps, Stevens se cantonnait encore à son rôle d'intendant et d'idéologue moderne, par qui le progrès devait se mettre au service des man-bush de Santo représentés par Buluk, et au-delà, à le faire partager à l'ensemble des Mélanésiens laissés pour compte du système colonial. L'idée de l'extension inter-insulaire du Nagriamel fut le déclencheur véritable de ses succès. Elle créa les circonstances favorables à une ascension solitaire de son leader, qui sut mettre ce mouvement à son service, alors qu'initialement prévalait le rapport inverse. L'administration française saisit cette occasion pour mener des négociations avec Stevens. Un accord tacite fut trouvé sur la question de la redistribution de terres en possession de la SFNH. Mais, au-delà d'un accord sur cette intention qui ne se traduisit pas dans les faits avant 1975, ces compromis instaurèrent une reconnaissance réciproque de l'autorité coloniale française et de celle décrétée coutumière de Stevens. La dimension traditionaliste du leadership de ce dernier s'en vit renforcée. Ces connivences avec l'administration française allaient graduellement s'intensifier en direction d'une pleine coopération, et entraînèrent « la transformation complète d'un mouvement de revendication foncière coutumier et isolationniste, en un parti politique francophile constitué sur des schémas occidentaux » (Beasant, 1984 : 31) [traduction de l'auteur].

Dans un premier temps, cet accord permit d'assurer la réussite de la politique d'immigration suivie par Stevens, en garantissant l'impunité de la reconquête foncière du Nagriamel et la neutralité intéressée des autorités françaises dans l'extension de la propagande de ce mouvement à d'autres communautés de l'archipel. Il rendait envisageable la constitution d'une fédération politique des îles du Nord sous la bannière du Nagriamel, d'une entité politique qui, forte de sa prétention à une légitimité coutumière, aurait eu l'extension d'une affiliation religieuse.

3. «Lors de ce meeting Jimmy Stevens et le chef Buluk furent intronisés comme les deux chefs commandant pour Santo. Stevens fit son allocution et précisa qu'il [...] entendait favoriser la coopération dans le travail avec tous, pour améliorer les conditions de vie à Vanuatu. À l'avenir, si quelqu'un devait avoir des problèmes fonciers, il devait voir Jimmy Stevens et le chef Buluk »(Van Trease, $1987: 140)$. 


\section{L'institutionnalisation du Nagriamel}

On assista progressivement à une concentration du pouvoir entre les mains de Jimmy Stevens. Auréolé de son récent prestige, ce dernier se passa progressivement de la caution indigène que représentait Buluk, en s'attribuant personnellement les attributs d'une légitimité coutumière. La revendication kastom dans le Nagriamel ne vise pas un retour vers le passé, mais elle représente bien plutôt, selon Van Trease (1987: 160), une force pour unir les néo-Hébridais dans une action positive contre la structure du pouvoir condominial, et une base à la construction d'un nouvel ordre socio-économique. Ces accumulations de contradictions se retrouvaient fréquemment dans les allocutions de Stevens :

«Le Nagriamel n'est pas un parti. Le Nagriamel n'est pas la politique. Il est le cœur des hommes, il est leur coutume. Mais pour la faire briller de nouveau [...] il faut utiliser la bonne méthode [...] le Nagriamel existait avant moi. Mais il faut le réformer de nouveau - pour s'en emparer et l'emblématiser sur le drapeau que nous hisserons. Voilà mon travail. Les gens me demandèrent - nous voulons voir le Nagriamel s'élever pour les générations à venir. Je leur répondis que je ferai de mon mieux... » (Stevens, in Van Trease, 1987 : 160) [traduction de l'auteur].

Van Trease précise que cette emphase portée sur la coutume, comme source de force et de revitalisation de la société mélanésienne, donna au Nagriamel sa pleine audience hors de Santo, et permit d'unir les partisans de Stevens une fois le problème des terres résolu, c'est-à-dire, une fois que les droits des Mélanésiens sur le bush inexploité furent reconnus. Fort de sa double légitimité administrative et kastom, Stevens inventa de toutes pièces une organisation sociale, économique et politique, pour régenter la vie communautaire à Vanafo. Un ordre traditionaliste devait fournir ses gages à un élan moderniste. Les observations de Hours, rapportées de Santo au début des années soixante-dix, offrent un précieux témoignage de la vie à Vanafo, aux heures de gloire du Nagriamel. La population y était estimée alors à cinq cent personnes. Le Nagriamel revendiquait, à cette époque, quinze mille adhérents pour tout l'archipel. La singularité de ce village et de l'ambiance qui y régnait est ainsi restituée par Hours :

«Les différentes populations de Vanafo nous mettent en présence d'un phénomène nouveau d'une certaine ampleur. Le caractère fédéral du mouvement se retrouve dans sa 'capitale', mais cette population variée si elle coopère ne se mélange qu'assez peu, sauf chez les jeunes. En effet, l'organisation en quartiers ou villages donne l'impression d'un assemblage dont chaque composante garde une certaine autonomie fondée sur l'île d'origine et ensuite sur l'appartenance religieuse qui est le second facteur de regroupement » (Hours, 1974 : 231).

Hormis certaines activités communautaires de la vie quotidienne, l'organisation collective de la production était essentiellement orientée autour des cultures commerciales et des activités de métayage. Le travail aux jardins traditionnels demeurait indépendant et s'accomplissait selon les pratiques en vigueur dans chacune des îles d'origines de leur exploitant. En 1977, d'après Van Trease (1987 : 163) :

« Il était clair que le Nagriamel représentait bien davantage que le simple produit de l'imagination de Jimmy Stevens. Il était matériellement fort bien organisé, comprenant différents projets agricoles... parmi lesquels l'exploitation forestière, l'huile de palme et la production d'arachide » [traduction de l'auteur].

Hours insiste sur l'organisation très formalisée des activités quotidiennes des villageois ${ }^{4}$. Mais l'efficacité de cette organisation de la production aurait été très faible, la participation étant inégale et épisodique. La répartition des bénéfices était égalitaire. Chaque exploitant d'une parcelle devait reverser la moitié des gains monétaires à l'« Union Labour Board Office », tout en conservant un droit de regard sur la redistribution ( $c f$. Van Trease, $1987: 164$ ).

«'L’Union Office' occupe un bâtiment [en dur, à Vanafo] faisant fonction d'hôtel de ville et de quartier général, au pied du mât porte-drapeau, centre politico-administratif du village, par opposition au centre traditionnel situé au pied d'un immense banian qui marque le centre public, le lieu de réunion des grands meetings. [...] [Il] est une sorte de ministère des

4. « La journée débute à six heures du matin par une levée des couleurs. Celle-ci est annoncée par des tambours d'Ambrym qui résonnent alentour. Le drapeau est hissé au sommet du mât devant un rassemblement d'hommes et de femmes alignés de part et d'autre dans une attitude proche du garde à vous. Suit un discours mobilisateur incitant au travail ou évoquant un problème actuel, ou encore visant à créer une émulation. Ce discours du secrétaire du comité est prolongé par des prières du responsable de l'église Church of Christ et d'un responsable presbytérien lorsqu'il est présent. Pendant cette cérémonie le public entonne l'hymne du Nagriamel.

De sept heures jusqu'à onze heures, chacun vaque à ses occupations ou se rend au travail commun, de même après la pause entre onze et treize heures. La journée se termine à seize heures et se poursuit par les bains à la rivière, les femmes s'y rendent à quinze heures, les hommes à dix-sept heures. Le panneau affiché mentionne la nuit à vingt-deux heures. Le rythme quotidien est marqué par l'appel d'une cloche lors des deux jours de travail communautaire. Le lundi en fin de journée un meeting réunit tous les villageois au pied du banian qui marque la place du village et le lieu des réunions publiques ouvertes » (Hours, 1974 : 233). 
travaux publics et du travail en général. Sa principale fonction est l'exploitation collective des terres consacrées aux productions agricoles commerciales [...]. L'Union possède un fichier de travailleurs qui exercent leur activité soit à l'intérieur du village, soit sous la forme de location de main-d'œuvre sur les plantations et dans les quelques entreprises de l'île » (Hours, $1974: 232$ ).

Stevens recevait une part sur les bénéfices laissés à l'appréciation de chacun des membres, une autre supportait l'organisation administrative du Nagriamel. Pour les membres du mouvement travaillant à l'extérieur de Vanafo, il fut fixé une retenue de $10 \%$ sur les salaires ( $c f$. Van Trease, 1987 : 165). Cet appareil administratif devait être complété par une banque, dont la construction n'a jamais dépassé le stade des fondations. Néanmoins, d'après Hours (1974 : 232) :

« Il faut voir dans cette organisation une structure en partie abstraite, d'une efficacité autant symbolique que réelle qui présente plus d'intérêt culturel ou idéologique qu'économique ».

La spécificité du Nagriamel est indissociable de la nature rationnelle de son leadership, c'està-dire de la manière dont Stevens conçut l'organisation administrative du mouvement pour conforter son pouvoir personnel. La hiérarchie exacerbée et le fédéralisme sont dès le départ deux fondements du mouvement. Derrière la position autocratique de Stevens se déroule tout un organigramme du mouvement obéissant à un ordre strict: «1/ Chief president (Jimmy Stevens) 2/ Assistant chief 3/ Land owner (Buluk) 4/ Chief Comitee 5/ Comitee members 6/ Secretary Comitee 7/ Union secretary " suivis de quelque trente qualifications d'importance décroissante (ibid.: 232). À chacune de ces qualifications correspondait un badge sur lequel était écrit le grade de son possesseur. Tous les man-bush de Santo étaient cependant tenus de s'appeler « frères ", et « amis » lorsqu'il s'agissait des autres insulaires du mouvement (ibid : 238).

Le comité était l'organe de délibération rassemblant les représentants de chaque île, pour discuter de la vie au quotidien comme des grandes lignes politiques du mouvement. Il se déroulait suivant un protocole moderniste avec secrétaire et prise de notes, et faisait également office de tribunal coutumier (ibid : 232) ; chaque insulaire était jugé selon les traditions de Santo. Par ailleurs, Van Trease souligne également, parmi les traits caractéristiques du Nagriamel, un subs- tantiel développement administratif. Ce comité se décomposa par la suite en plusieurs organes de délibérations. Le « Land Comitee » était l'institution la plus englobante. Composé de vingt et un chefs coutumiers de Santo, il fixait les règles du mouvement à l'égard des colons et des investisseurs étrangers sur les terres indigènes ( $c f$. Van Trease, 1987 : 162). Le « Upper Comitee » comportait pour sa part quinze membres provenant des quinze îles que regroupaient le Nagriamel. Leur fonction était de superviser l'application des projets approuvés par le Land Comitee. L'instance administrative supérieure, le «Ten Head Comitee », détient le pouvoir exécutif, il ne comprend que des membres reconnus aux rangs de "chefs coutumiers" ayant vécu au moins cinq ans à Vanafo. Toute décision concernant la communauté de Vanafo était d'ailleurs du ressort de cette instance. Mais dans tous les cas de figures, comme le rappelle Hours (1974 : 232) :

« Les grandes options sont prises par le chef président et discutées en comité le cas échéant ».

\section{Les soutiens extérieurs du Nagriamel}

Les alliances extérieures du Nagriamel furent mises en place à la seule initiative de Stevens. Elles renforcèrent sa dérive autocratique sur le mouvement, accentuèrent le caractère politique et idéologique moderne de ses moyens d'actions et, par extension, provoquèrent sa fuite en avant vers des menées séparatistes. Les premiers contacts extérieurs du Nagriamel furent pris avec André Leconte, un millionnaire calédonien soucieux d'agrandir une de ses plantations à Santo. L'arrangement conclu par les deux parties portait sur l'attribution d'un hectare de terre à Leconte pour chaque heure d'utilisation par les gens de Vanafo des équipements mécanisés appartenant à ce dernier. Ce premier rapprochement dévoila une des nombreuses ambiguïtés de la stratégie de Stevens : combattre pour la protection de la tenure foncière indigène, tout en facilitant l'exploitation de ces mêmes terres par des investisseurs étrangers. L'alliance du Nagriamel avec Leconte fut également un test pour l'administration française dans sa stratégie de redistribution foncière et de reconquête de l'estime des Mélanésiens, par le développement des infrastructures et des administrations francophones ${ }^{5}$. L'accord ne se matérialisera cependant pas, Leconte abandonna la partie au cours de l'année 1970, et le profit rapporté par cette

5. Après 1974, la redistribution des terres s'accéléra ; 8800 hectares furent ainsi rendus jusqu'à la fin des années soixantedix. La construction de l'école et du dispensaire français à Vanafo a été réalisée de 1973 à 1974 ( $c f$. Van Trease, 1987 : 149). Une route traversant l'île jusqu'au nord fut également ouverte de Vanafo à Matantas. 
alliance ne fut pas celui escompté. Par contrecoup, Stevens fut confronté aux premières dissensions dans son mouvement, ce qui ne l'empêcha pas de réitérer avec force ce type d'alliances ${ }^{6}$.

Cette période correspond également pour le Nagriamel aux premières tentatives de Stevens d'adopter une attitude militante sur un plan international. En 1969, les premiers contacts furent pris entre Stevens et les nationalistes fidjiens, qui jugèrent le personnage peu crédible. Toutefois, l'intermédiaire par lequel avaient été établis ces contacts, l'avocat fidjien Karam Ramkhara, poursuivit pour le compte du Nagriamel des démarches auprès de l'ONU et y présenta en 1971 une pétition au comité de décolonisation, condamnant le Condominium et requérant une enquête sur les velléités d'indépendance à Santo. Stevens justifia cette démarche au Fiji Times: «Les indigènes se font emprisonner et, dans de nombreux cas, ils se voient menacés et chassés de leur terres » (in Van Trease, 1987 : 149). Stevens avait également pris, dès 1967, des contacts avec Eugène Peacock, un spéculateur terrien américain désireux d'investir aux Nouvelles-Hébrides. Ce fut là, une des alliances les plus retentissantes du Nagriamel. Peacock, commercialement basé à Hawaii, opérait sur une vaste échelle avec de gros moyens. Il avait acquis d'immenses superficies de terres, notamment à Santo. Son plan était de subdiviser ses propriétés en petites parcelles pour les revendre à des anciens combattants américains du Vietnam (cf. Beasant, 1984 : 45).

Au cours des années soixante-dix, les responsables du Nagriamel devinrent préoccupés par la concurrence exercée par le très récent National Party (NHNP futur Vanuaaku Pati) dans le domaine des revendications foncières ${ }^{7}$. Stevens fut convaincu de jouer la surenchère et d'adopter une démarche de confrontation avec le Condominium, pour accélérer la redistribution des terres et accroître les aides au développement en faveur du Nagriamel. De son côté, Peacock subit un revers majeur lorsque les autorités condominiales décidèrent de faire voter deux lois rétroactives sur l'indivision des terres et sur une hausse très importante des taxes sur l'exploitation commerciale de parcelles vierges. Ces lois avaient explicitement pour but de briser les manœuvres de Peacock, mais aussi de faire un exemple et d'atténuer les effets de la loi condominiale qui avait accordé en 1971, aux Nouvelles-Hébrides, le statut de " paradis fiscal ${ }^{8}$. La volonté de ce dernier de combattre ces décisions, le conduisit à développer, par le biais de son associé JeanJacques Hénin, la dimension politique d'une collaboration avec le Nagriamel. Au plan économique, celle-ci s'était traduite par un accord sur les activités de la SODEPAC, une station d'élevage doublée d'une compagnie de déboisement appartenant à Peacock, située à six kilomètres de Vanafo, pour laquelle les membres du Nagriamel fournissaient la main-d'œuvre.

La requête du National Party à l'onu, en 1974, en faveur d'une indépendance en 1977, mit le feu aux poudres. Elle décida de l'alliance du Nagriamel avec le parti des colons français de Santo - le Mouvement Autonome des NouvellesHébrides (MANH) - et de ses rapprochements avec le mouvement John Frum de Tanna, l'Union des Communautés des NouvellesHébrides (UCNH) et le parti francophone de Port-Vila. Cette coalition des partis appelés par la suite les Modérés, acheva de transformer le Nagriamel en un mouvement politique antinationaliste, anti-anglophile et cryptocolonialiste, puisque s'alignant sur les positions du MANH, il ne contesta même plus le droit des colons à exploiter la terre de Santo ( $c f$. Beasant, 1984 : 28). Peacock, déstabilisé par une bataille juridique qu'il perdit contre les autorités du Condominium, misa tous ses espoirs en Stevens et le Nagriamel. L'idée était de constituer une force nationaliste favorable aux entreprises spéculatives de Peacock - ou selon les mots de Plant (1977 : 51) « a 'native faction' with the right philosophy » -, en incitant Stevens à durcir la stratégie de confrontation avec le Condominium. Cette collaboration fut totale, et d'importants transferts de fonds furent opérés en faveur du Nagriamel. Jean-Jacques Hénin fut pour sa part nommé « Conseiller en développement » du

6. Des dissensions plus importantes apparaîtront en 1974 lors de l'officialisation de la démarche de Stevens et de la signature d'une alliance électorale avec le Mouvement Autonomiste des Nouvelles-Hébrides (MANH). Naîtrons en réaction à ses compromissions deux petits partis qui demeurèrent marginaux le Natuito Party («Children of the land») qui deviendra l'appendice coutumier du futur Vanuaaku Pati à Santo, et le Tabwemassana (du nom du plus haut pic de Santo) créer par un planteur français pour contrer justement le Natuitano ( $c f$. Beasant, 1984 : 30-31).

7. L'opposition du Nagriamel, indique Hours (1974:238), fut celle « des gens de la brousse par opposition aux indigènes les plus évolués, anglicans, scolarisés, employés ou petits fonctionnaires urbains, petits propriétaires, que sont les 'cols blancs mélanésiens' et l'amorce d'une petite bourgeoisie indigène politisée ».

8. Plant rappelle dans son article que Santo était devenu dès ce moment une véritable Mecque pour spéculateurs. Il présente quelques commentaires extraits de la presse de l'époque : les Nouvelles-Hébrides y figurent comme « le dernier Paradis », «le plus récent et le plus sûr des Paradis Fiscaux », « if you want laisser faire capitalism go to the New Hebrides » (Plant, 1977 : 49-50). 
Nagriamel. Quant à Peacock, il reçut de Stevens les pouvoirs de "Chef financier» du mouvement (cf. Beasant, $1984: 48-49$ ).

L'échec des Modérés aux premières élections générales de novembre 1975 et la piètre performance de l'alliance entre le MANH et le Nagriamel sur Santo, amenèrent Peacock à abandonner ses dernières illusions, malgré sa participation à l'organisation de plusieurs démonstrations de force à Luganville en décembre de la même année. Celles-ci se solderont d'ailleurs par l'expulsion administrative de Peacock hors des Nouvelles-Hébrides. Quant à Stevens, ces vicissitudes le poussèrent à embrayer le pas vers des menées toujours plus séparatistes. Ce n'est que tardivement, au moment même où se déroulaient les premières manifestations violentes à Luganville, que les autorités coloniales réalisèrent que parmi les soutiens étrangers les plus résolus à la cause de Stevens ne se trouvait pas le seul Peacock. L'aide la plus active au Nagriamel provenait, depuis le courant de l'année 1975, du milliardaire américain Michael Oliver et de sa Phœnix Foundation ${ }^{9}$.

L'influence de Oliver sur Stevens et le soutien logistique apporté par la Phœnix Foundation permirent la constitution d'une Fédération des Communautés Auto-Gouvernées du Nagriamel le 27 décembre 1975, et à programmer l'indépendance des îles du Nord. Cette fédération était censée inclure Santo et ses îlots, excepté le centre urbain de Luganville; toutes les îles voisines, Aore, Malo, Aoba, et Maewo; toutes les îles appartenant au groupe des Banks et des Torres ; ainsi que tous les autres groupements des Nouvelles-Hébrides qui désiraient se joindre à la fédération, en tant que peuple libre et indépendant. Stevens avait préparé dans ce sens, avec l'aide des juristes de la Phœnix Foundation, une Constitution ${ }^{10}$, et reçut également de cette dernière tous les symboles matériels d'un État souverain. Une monnaie fut frappée, des passeports et des constitutions imprimés, un drapeau fut choisi. Radio Vanafo reçut des moyens techniques et une assistance pour étendre sa diffusion. Enfin, les transferts de fonds pour développer la structure administrative du Nagriamel et créer une banque fédérale, se firent plus substantiels.

Le Nagriamel différera à deux reprises sa déclaration d'indépendance, avant que cette revendication ne soit mise en veille jusqu'aux événements de 1980. Oliver fut, à son tour, l'objet d'une sanction d'expulsion, mais poursuivit son aide active au Nagriamel, dans la perspective de mener la sécession de Santo à son terme. Comme le souligne très justement Beasant (1984: 51) :

« Si les déchaînements du Nagriamel ont constitué le point culminant des manœuvres politiques de Peacock, elles ne furent qu'un point de départ dans les tentatives d'Oliver et de la Phœnix Foundation pour faire de Santo un État indépendant » [traduction de l'auteur].

\section{Vemarana versus Vanuatu : le Nagriamel et la sécession de Santo}

Dans la période de transition qui devait mener les Nouvelles-Hébrides à l'indépendance, la France encouragea l'hypothèse d'une structure largement confédérale de la future République. Celle-ci devait laisser ouverte la voie d'une étroite association de cet archipel avec la Nouvelle-Calédonie, afin d'éviter tout effet de contamination indépendantiste sur les autres territoires du Pacifique, et garantir ainsi le pro-

9. Moses Olitsky de son vrai nom ( $c f$. Van Trease, $1987: 149$ ), était un juif lituanien, rescapé des camps Nazis, qui fit fortune au Etats-Unis, et mit celle-ci à contribution pour son indéfectible cause : la création d'un Etat associant des principes idéologiques libertaires et l'ultra-libéralisme de l'économie de marché. Car pour lui, les Etats-Unis des années soixante-dix étaient définitivement devenus un « Etat socialiste fasciste ». Avant qu'il ne s'intéresse au cas de Santo au Nagriamel, il tenta par deux fois de s'emparer d'une île pour y fonder un nouvel Etat où seraient mises en application les dispositions légales contenues dans son ouvrage publié en 1966 sous le titre « New Constitution for a New Country ». Sa première tentative prit la forme de la création d'une "République de Minerve » sur un récif corallien appartenant au Royaume de Tonga. La seconde consista à diligenter une sécession de l'île d'Abaco aux Bahamas. Mais pour Oliver, à Santo, les chances de succès devenaient sérieuses : «En comparaison avec Abaco, nous avons trouvé les gens du Nagriamel exceptionnellement bien organisés » (Oliver, in Plant, 1977 : 54) [traduction de l'auteur].

10. «La Constitution se présente sous la forme d'un petit document de sept pages. La couverture reproduit l'emblème du mouvement : une main noire et une main blanche serrées, au-dessus desquelles figure un pentacle surmonté du bouquet de feuilles de namele et de nangaria. L'intitulé s'énonce ainsi : 'Constitution of Confederation of Natakaro (Formerly New Hebrides Islands)'. Elle est composée de neuf articles, ratifiés de la main même de Jimmy Stevens, avec le cachet du 'Nagriamel Chief President Union Council'. Le texte précise que chaque groupement (unité géographique et sociale) détient le contrôle de son propre territoire, l'autorité sur ses propres lois, ses coutumes et ses traditions. Le gouvernement de la Fédération est réduit aux tâches essentielles qui sont de protéger et de défendre la vie et les droits de chacun. Des procédures électorales par groupement désignent les membres de la chambre supérieure, le Sénat, et de la chambre inférieure, le Nasara (nom dans les îles du Nord pour désigner la 'maison des hommes initiés'). Le pouvoir exécutif est assuré par le Chef-Président de la Fédération Nagriamel. L'organisation militaire est subordonnée au pouvoir civil. Enfin, la déclaration des droits insiste sur la défense de la vie et de toutes les libertés (de conscience, de choix religieux, de parole et de presse, de réunion et de revendication » (Bernard, $1983: 52)$. 
gramme d'expérimentations nucléaires français en Polynésie. Parmi les sympathisants du Vanuaaku Pati, les autorités françaises furent soupçonnées, dans leur insistance sur l'idée d'une confédération, d'encourager une possible sécession de Santo ${ }^{11}$. Pour le moins, les autorités françaises furent fidèlement informées des plans de Stevens, puisque lors de sa visite à Paris au premier trimestre de 1980 en compagnie de Michael Oliver chez qui il venait de séjourner à Carson City, Stevens offrit à Paul Dijoud, Secrétaire d'État aux DOM-том, les gages d'une association de Santo avec la Nouvelle-Calédonie et lui remit un exemplaire de la constitution de Natakaro signifiant une partition des quinze îles du Nord des Nouvelles-Hébrides ( $c f$. Beasant, $1984: 68$ ).

La Phœnix Foundation continua pour sa part à participer de loin aux menées séparatistes du Nagriamel, en l'intégrant dans un réseau d'influences internationales autour du cas de Santo, en finançant l'encadrement administratif du Nagriamel. La manifestation la plus médiatique de cette collaboration fut le plan monté par Oliver pour transférer, sous l'égide de l'organisation Fatima International, 100000 boat people vietnamiens à Santo. Cette proposition donna lieu à une conférence de presse à Sydney en janvier 1979, et fut confirmée dans le même temps par Stevens sur Radio Vanafo. Dans ce fouillis d'alliances tacites et de collaborations déclarées, de spéculations diverses, d'entreprises coloniales sans véritable coordination, les élections de 1979 viendront faire l'effet d'un coup de pied dans la fourmilière ${ }^{12}$. Les alliés du Nagriamel furent mis devant le fait accompli quant à l'échec de leur stratégie légaliste. Les autorités françaises elles-mêmes en vinrent dès ce moment à s'accorder sur un double langage, acceptant la perspective d'une indépendance sous l'autorité d'élites anglophones, tout en laissant ouvertes les solutions les plus extrêmes dans l'intention de préserver toutes sortes d'intérêts, plus ou moins incompatibles (ceux des petits colons à court terme, de la position de la France dans le Pacifique à moyen terme et du maintien de la francophonie à long terme).

La perte de ces élections provoqua un véritable choc dans le camp francophone. La situation se dégrada très vite. Dans les heures qui suivirent son annonce, les premières réactions se firent entendre à Santo : Jimmy Stevens déclara sur Radio Vanafo que les élections avaient été truquées et proféra un certain nombre de menaces à l'intention des membres du Vanuaaku Pati résidant à Santo. Les démonstrations de force et les intimidations du Nagriamel et du parti Tabwemassana envers leurs opposants politiques se déchaînèrent. Les francophones et leurs infrastructures furent totalement épargnés des saccages que subirent les antennes administratives et les locaux commerciaux des anglophones.

Tout envoi de troupes fut bloqué par l'inspecteur-général Robert, contre l'avis des britanniques et de leurs protégés du VP. Cette opposition des autorités françaises à toute intervention armée sur Santo persistera durant les six mois suivants, jusqu'à la veille de l'indépendance du pays en juillet 1980. Toutes les discussions et les tentatives de conciliation qui se succédèrent, semaines après semaines, échouèrent. Stevens évita autant qu'il put ces négociations (bien qu'il accompagna fin février la délégation des partis Modérés à Paris) ( $c f$. Beasant, $1984: 84$ ) et déclara, dès janvier 1980, que la voie d'une indépendance séparée pour les îles de Santo et Tanna était désormais un fait irréversible. Le 21 janvier, il somma l'administration britannique d'interrompre ses activités à Santo et donna sept jours à ses fonctionnaires pour quitter l'île.

La réplique du gouvernement central à la proclamation d'un gouvernement de la république de Vemarana sur Santo fin janvier (ibid: 82), se fit attendre jusqu'à début mai, et prit la forme d'une décision du conseil des ministres fixant le 30 juillet 1980 comme date ultime pour l'indé-

11. Cette éventualité d'une sécession de l'île de Santo, ou de toute autre île de l'archipel des Nouvelles-Hébrides ne fut officiellement jamais admise par le gouvernement français et ses représentants. Toutefois, les affirmations contradictoires des deux principaux responsables français présents aux Nouvelles-Hébrides durant la rébellion, à savoir Jean-Jacques Robert et Yves Rodrigue, respectivement délégué extraordinaire et ministre plénipotentiaire, ne font que renforcer les doutes sur la position officieuse de la France. D'après Jean-Jacques Robert, le gouvernement français s'est toujours tenu à la perspective d'une unité territoriale indivisible de l'archipel, bien qu'il admette l'effectivité des tentatives de déstabilisation du gouvernement indépendant de Vanuatu et de manipulations des colons de Santo de la part des autorités de Nouméa sur lesquels lui-même n'aurait eu aucun contrôle. En revanche, Yves Rodrigue admet pour sa part l'intervention des services secrets français dans l'organisation même de la rébellion de Santo et du déclenchement des violences sur Tanna, dans le but de faire diversion et de négocier un avenir français pour Santo et son intégration à la Nouvelle Calédonie, en lâchant Tanna en échange (propos recueillis lors d'une série d'entretiens qui me furent accordés par les intéressés à Paris en novembre 1997 et mai 1998).

12. Une constitution non consensuelle avait été adoptée en octobre 1978 par un gouvernement d'union nationale dont le VP s'était volontairement exclu dans l'attente d'une consultation populaire. Or, le 16 novembre 1979, lorsque furent connus les résultats des élections qui s'étaient tenues deux jours plus tôt, il apparut que non seulement le VP avait remporté la victoire, mais encore que celle-ci fut d'une ampleur insoupçonnée : 26 des 39 sièges de la nouvelle assemblée revenaient au VP, soit un total de $62,3 \%$ des voix ; une majorité des $2 / 3$ qui lui conférait le privilège de changer la Constitution. 
pendance. Cette décision engendra en retour, dans la nuit du 27 mai, le saccage du centre administratif britannique, des prises d'otages et le vol de munitions et de dynamite par les sympathisants du Nagriamel. En cette occasion, ils s'emparèrent de la municipalité de Luganville. Cette nuit agitée fut suivie au petit matin par un discours de Stevens sur Radio Vanafo rebaptisée, entre temps, du nom de Radio Vemarana :

« Ceci est la voie de la délivrance et de la liberté, protégée et défendue par le gouvernement de la Fédération Indépendante du Nagriamel, Santo, NouvellesHébrides. Ceci est le service de radiodiffusion situé approximativement à $25^{\circ}$ de latitude sud et $168^{\circ}$ de longitude ouest de la planète terre ou Urantia, sous les limbes de la Voie lactée. Aujourd'hui est né le Vemarana. Les gens de Santo lui ont donné ce nom et les gens de Santo travaillent pour son administration. Venez rejoindre le Vemarana, quelle que soit votre race, pour tous ensemble aider le gouvernement de Vemarana à quitter le pays de Vanuatu. Le Nagriamel fut indépendant dès 1976, alors que, si nous avons bien compris, l'indépendance du gouvernement de Vanuatu ne prendra effet qu'en juillet 1980. Soyez vigilants, car le Vanuatu indépendant n'est pas la même chose que le Vemerana indépendant » (Stevens, ibid : 94).

\section{La « guerre des cocotiers » et ses conséquences}

Dans les jours qui suivirent, le gouvernement élu de Vanuatu décréta un blocus total de Santo, décision qui entraîna le départ de l'île de 2000 résidents britanniques et mélanésiens anglophones. Le 2 juin, fut composé un Cabinet du Vemarana. Le 6 juin, Stevens ordonna la libération de tous ses otages. Durant tout le mois, la capitale, Port-Vila, connut à son tour une aggravation des tensions, suite à une grande manifestation des francophones. Divers signes d'émergence d'une dissidence violente furent relevés, parmi lesquels l'apparition, sur les murs de la capitale, de graffitis O.A.S. Cette situation entraîna l'arrivée en renfort, le 15 juin à Port-Vila, de 200 marines anglais, après que la France envoya 55 gardes mobiles. Ces manœuvres renforcèrent un climat devenu anxiogène. Les jours précédents avaient en effet vu éclater de nouveaux troubles à Tanna, en juin, marqués par l'assassinat de Yolou ${ }^{13}$.

À Santo par contre, le Vemarana connut deux mois d'indépendance effective; les administrations furent réouvertes, les fonctionnaires payés, et le blocus largement contourné grâce à une logistique assurée par ses nombreux soutiens néo-calédoniens. Mais avec l'approche de la date fatidique et sous la pression des manifestations du VP soutenue par les Britanniques, il fut exigé par Andrew Stuart, délégué de la Reine d'Angleterre, que les délais pour une indépendance le 30 juillet soient tenus. Le 14 juin, Walter Lini assista, en tant que chef du gouvernement légitime, au Forum du Pacifique Sud à Tarawa (Kiribati) et signa le 17 avec les autorités de PNG un accord sur une intervention de leurs troupes à Santo pour y réduire la sécession.

Devant la tournure prise par les événements, l'intransigeance des colons de Santo et la détermination du Nagriamel, les autorités françaises réalisèrent que toute tentative de sécession était désormais vaine. Aussi, Britanniques et Français s'entendirent sur un rétablissement de l'ordre à Santo avant le 30 juillet, au moyen d'un contingent mixte de 100 marines et de 100 parachutistes, même si les Français justifiaient leur décision au nom de la seule protection de leurs ressortissants et se refusaient à envisager une quelconque répression. L'envoi à Luganville d'une troupe conjointe, placée sous commandement français, eut lieu le 24 juillet. La veille, l'inspecteurgénéral Robert s'était rendu à Santo pour y tenir un discours qui, tout en se voulant rassurant, contribua par la suite à insuffler un climat de panique, lorsque trois semaines plus tard l'échec patent de cette intervention entraîna le départ des troupes franco-britanniques et l'arrivée consécutive des troupes papoues ${ }^{14}$.

La Kumul Force, composée de 300 hommes, deux navires patrouilleurs, et quatre avions ( $c f$. Bernard, 1983 : 117), prit position sur Santo le 18 août. Le premier jour de l'intervention, le transfert de pouvoir entre les mains du Commandant papou, le Colonel Toy Huai, se déroula sans heurts, les troupes papous étant occupées à

13. La mort de Yolou fut considérée comme une perte irrémédiable par le camp francophone. Jean-Jacques Robert, pour qui il ne fait aucun doute que Yolou fut délibérément tué par un militant VP, conteste le fait que la France ait eu une quelconque intention de profiter de ce meurtre pour renverser le gouvernement de Walter Lini ( $c f$. Paitel, 1985). Par contre, l'autorisation lui fut refusée par son ministre de tutelle, Paul Dijoud, de faire exécuter en représailles une action du même ordre (entrevue personnelle, mai 1997). Plus officiellement, Robert déclare : « Alexis Yolou avait fait des études supérieures en France [quelques semaines à l'École de France d'Outre-mer] ; ses capacités devaient lui permettre de jouer un rôle de premier plan dans l'histoire de son pays. Son meurtre souleva une réprobation unanime. Le refus du Procureur Mac Kay, un anglais, chargé de l'enquête, de poursuivre les coupables au motif du manque de preuves, déchaîna la fureur des amis de la victime et des 'coutumiers' de Tanna. Devant ce déni de justice, dans une région où la loi du talion n'avait pas encore complètement disparu, certains estimèrent que pour rétablir l'équilibre, un dignitaire VP, presbytérien de préférence, devait être sacrifié. Etant en charge, alors, de la Résidence de France, je réussis [...] après avoir hésité longtemps, à leur faire abandonner leur projet » (Robert, nd. : 6).

14. Pour une retranscription du texte complet de l'allocution de Jean-Jacques Robert, voir Paitel (1985: 234-235). 
s'emparer des points stratégiques de Luganville. Mais dès le lendemain, les contrôles et les arrestations commencèrent. A partir du 23 août, un certain nombre d'actes de sabotages furent commis par des colons ayant pris le maquis, ce qui accentua la répression et offrit le prétexte à la venue de renforts papous, composés de commandos spécialement entraînés pour le combat dans la jungle ( $c f$. Beasant, 1984 : 130). Le 30 août, le fils aîné de Stevens fut abattu par les Papous lors de la fusillade du véhicule dans lequel il circulait en compagnie de deux colons.

Le lendemain, les troupes papoues investirent Vanafo et Port-Olry (autre bastion de la résistance). Ils y trouvèrent Stevens, assis sur une chaise sous le grand banian du village, avec derrière lui, un regroupement de quelques centaines de man-bush, hommes, femmes et enfants. Parmi les quelques Européens encore présents à Vanafo où ils avaient trouvé refuge, certains tentèrent de s'échapper ou de se défendre contre les soldats, mais la plupart en furent empêchés. Quelques centaines de Vémaranistes furent arrêtés durant ces opérations, et malgré l'arrestation de Jimmy Stevens, des accrochages avec les forces papoues se poursuivirent dans le Nord de Santo pendant quelques jours.

De septembre à novembre, la répression se prolongea sous l'action conjointe des forces papoues, de la Vanuatu Mobile Force (constituée pour l'essentiel de miliciens du VP) et des avions de la Royal Australian Air Force assurant le transport des prisonniers vers Port-Vila. De nombreuses brutalités furent constatées et beaucoup d'arrestations eurent un caractère sommaire, malgré diverses protestations dont celles du Conseil des Églises de Vanuatu (Vanuatu Christian Council) et d'Amnesty International (cf. Bernard, 1983 : 122-125, 179-181). Le chiffre officiel donné par le gouvernement de Vanuatu sur le nombre d'arrestations, était de 2774 (ibid: 125). Les peines les plus fortes allaient de cinq à sept ans d'emprisonnement. Quelques centaines de Français échappèrent à toute poursuite judiciaire en fuyant vers la Nouvelle-Calédonie et 127 ressortissants étrangers furent déclarés interdits de séjour, dont 110 citoyens français ( $c f$. Beasant, 1984: 143). Les lois condominiales n'ayant pas prévu le délit de haute trahison, onze chefs d'inculpation furent prononcés contre Jimmy Stevens le 21 novembre par le juge britan- nique Cook ${ }^{15}$. Les condamnations donnèrent lieu, illégalement, à une accumulation des peines. Stevens, assumant durant le procès toute la responsabilité de la rébellion, se vit infliger quatorze ans et demi de prison et 220000 vatus d'amende. Pour sa défense, il déclara :

« Je suis un des grands chefs de la coutume du Nagriamel et je voudrais que le Président de la Cour me considère en tant que tel. Parce que des gens m'ont demandé de remplir des fonctions, je les ai acceptées (parce que mon peuple m'a demandé de faire des choses, je les ai faites). Je n'avais pas envisagé tous les troubles qui pouvaient avoir lieu. Aujourd'hui, je peux voir tout le mal qui a été fait et je réalise que j’ai fait une erreur en acceptant le rôle de Premier ministre du Vemarana. J'ai écouté bien trop de gens, les représentants de la France avaient promis de ne jamais me laisser tomber, l'Inspecteur Général Robert me l'avait promis. Ils me l'avaient tous promis... Cela fait vingt ans que je dirige le Nagriamel, il n'y a jamais eu de tués par ma faute, mais le Vanuatu a tué trois de mes hommes : Alexis Boulouk, Alexis Youlou à Tanna, et l'un de mes fils, il y a peu de temps. C'est tout ce que j'avais à dire » (Stevens, in Bernard, 1983 : 125-126).

\section{Le Nagriamel et l'indépendance nationale}

Après près de onze ans d'incarcération, Jimmy Stevens, très malade, fut libéré prématurément en 1991, juste avant le premier changement de majorité intervenu dans l'histoire récente de Vanuatu. En effet, Walter Lini, avant sa destitution le 6 septembre 1991 sur l'initiative de son propre parti, avait pris la décision de libérer Stevens trois mois avant les élections générales, en contrepartie d'une « réconciliation coutumière » entre les deux hommes ( $c f$. Henninghman, 1992 : 404-405), et de jeter ainsi un trouble dans l'opposition. Mais gravement malade, Stevens s'éteignit en 1994, laissant le Nagriamel dans l'état dans lequel il s'était trouvé depuis la fin précipitée de la rébellion sur Santo.

Le Nagriamel ne fut jamais interdit, mais fit l'objet d'une constante surveillance, bien que tous ses soutiens étrangers se soient définitivement évanouis. Vanafo fut considéré durant tout le règne de Walter Lini comme un village de dissidents. Pourtant le Nagriamel avait, depuis lors, renoué avec une attitude légaliste, participant aux élections de 1983 et 1987, où il obtint des scores marginaux à l'échelle de l'archipel, mais qui demeuraient substantiels à Santo. Le

15. Jimmy Stevens plaida coupable pour les onze chefs d'inculpation qui lui avaient été signifiés par le procureur général. À savoir : 1/ action militaire avec usage d'armes ; 2/ délégation d'hommes pour endommager le british Paddock ; 3/ détention arbitraire de délégués et de policiers ; 4/ détention d'objets volés : dynamite, munitions...; 5/ organisation d'un mouvement illégal ; 6/ incitation au non respect de la loi ; $7 /$ production illégale de passeports ; $8 /$ reproduction illégale de constitution ; 9 / financement d'une association illégale ; 10/ conduite préjudiciable à l'ordre public ; 11/ maintien et contrôle d'une radio illégale (Paitel, $1985: 262-263$ ). 


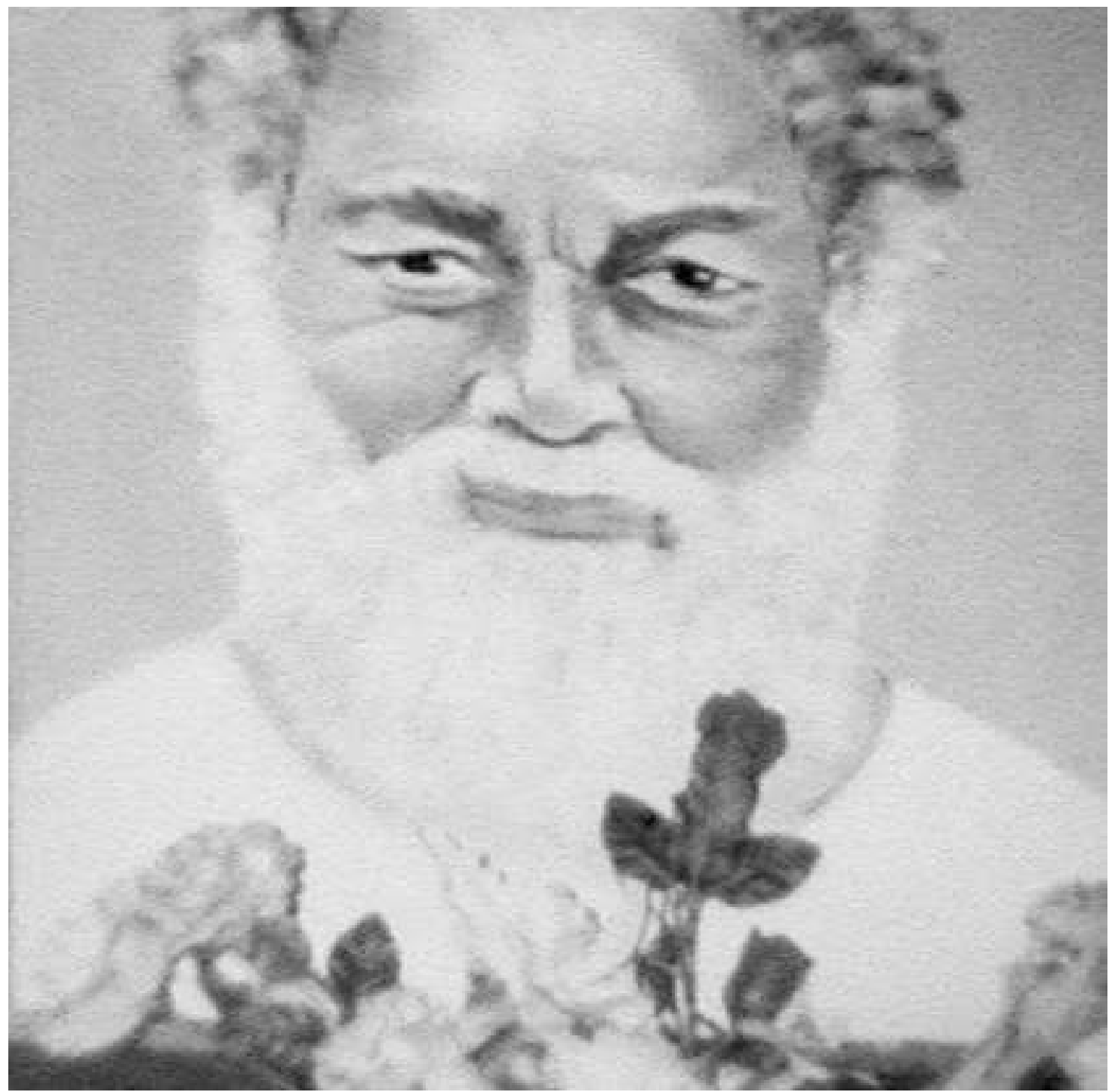

FIG. 1. - Portrait mortuaire de Jimmy Stevens. (C) M.K.Tabani. 


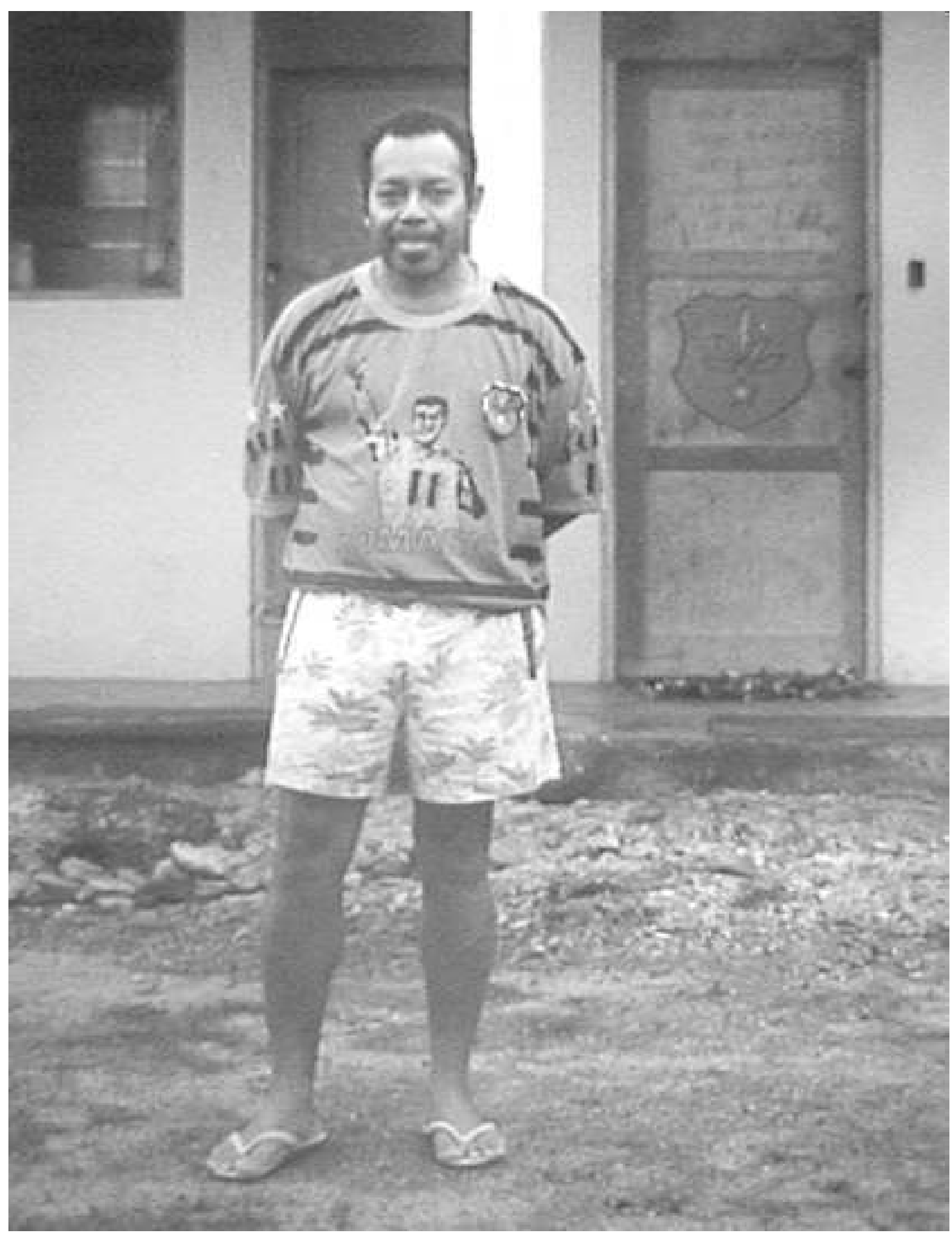

FIG. 2. - Franky Stevens, fils cadet de Jimmy Stevens. (C) M.K.Tabani. 


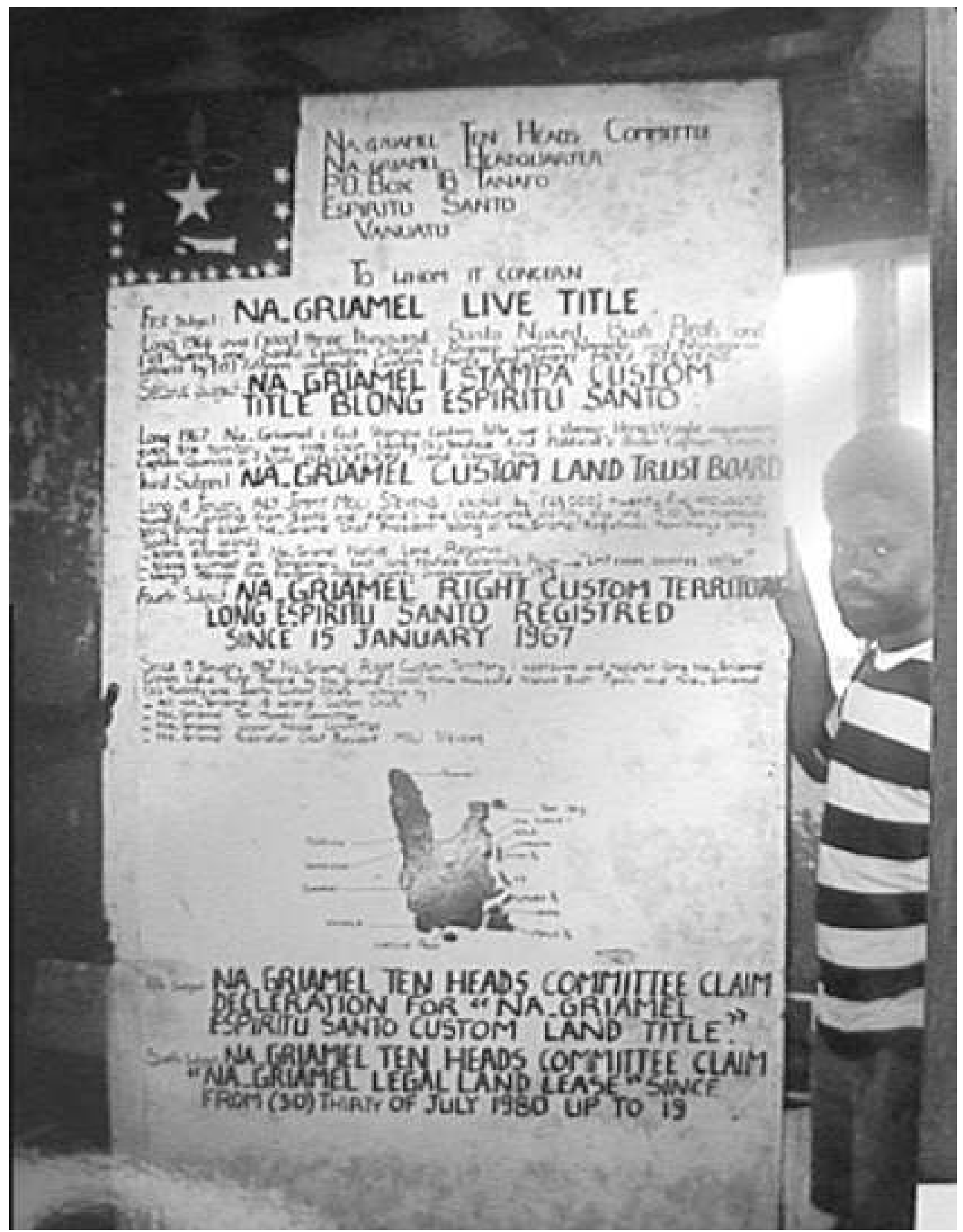

FIG. 3. - Panneau de propagande du Nagriamel. (C) M.K.Tabani. 


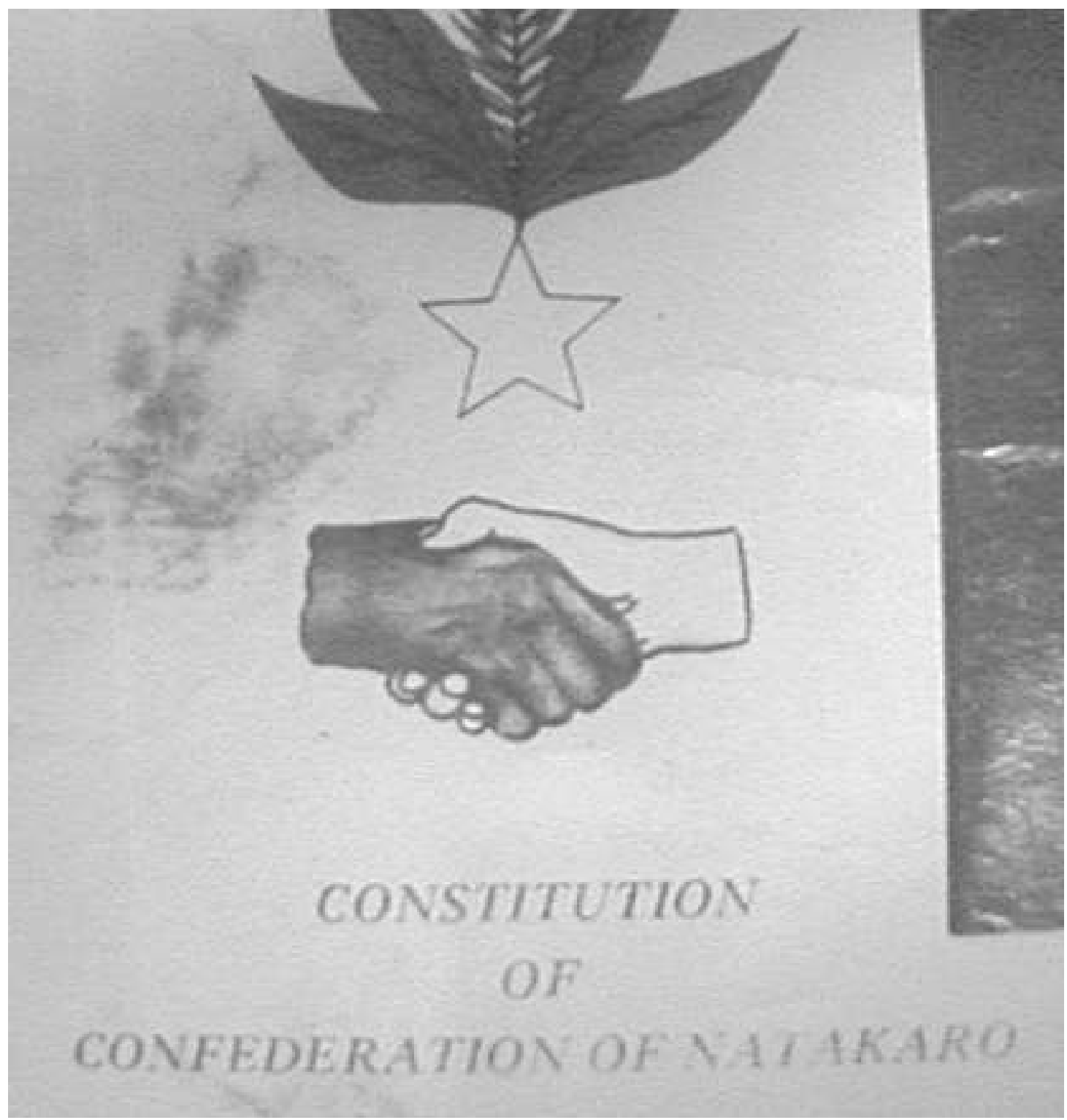

FIG. 4. - Constitution de Natakaro. (C) M.K.Tabani. 


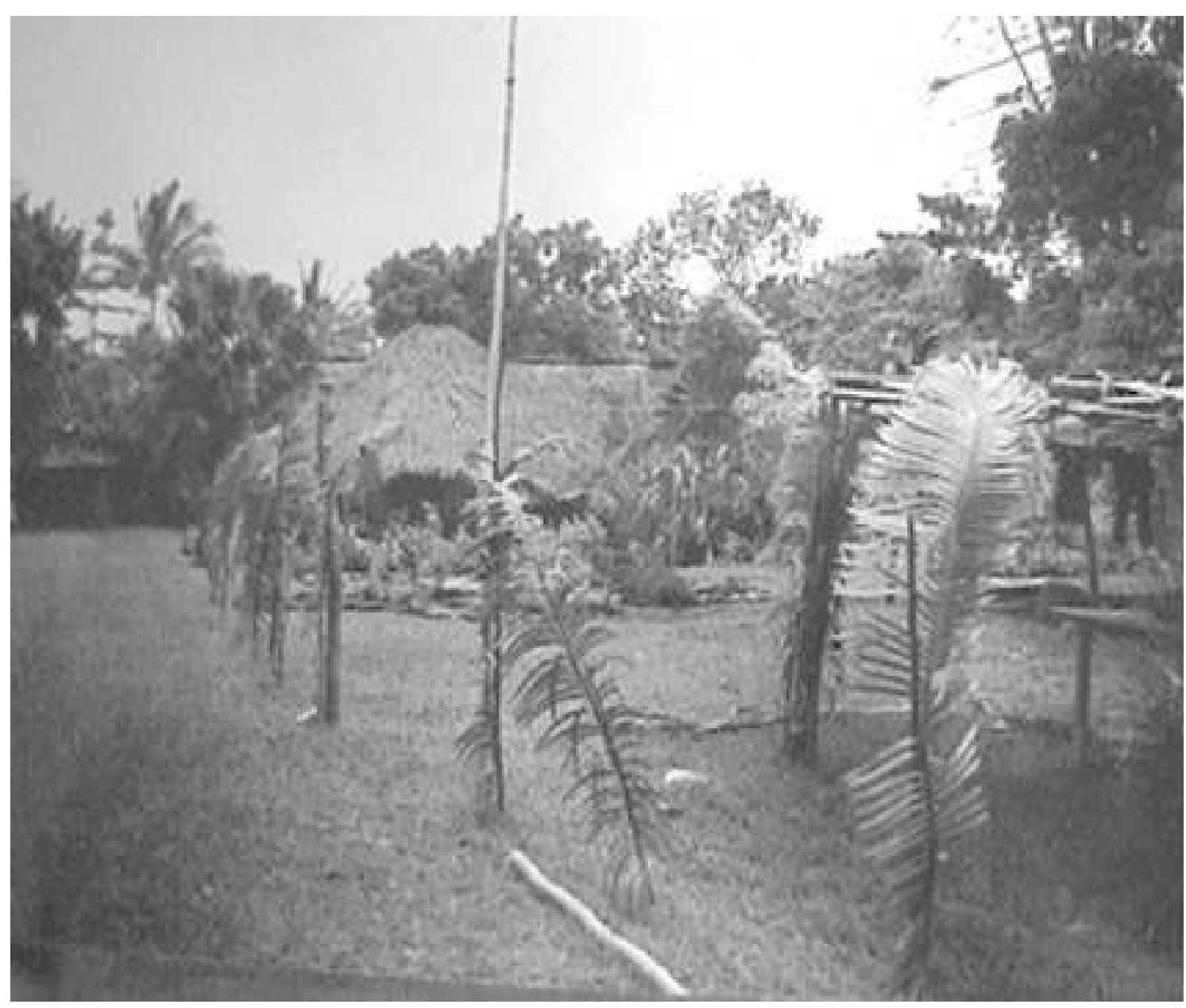

FIG. 5. - Ruines de Radio Vemarana à Vanafo. (C) M.K.Tabani. 


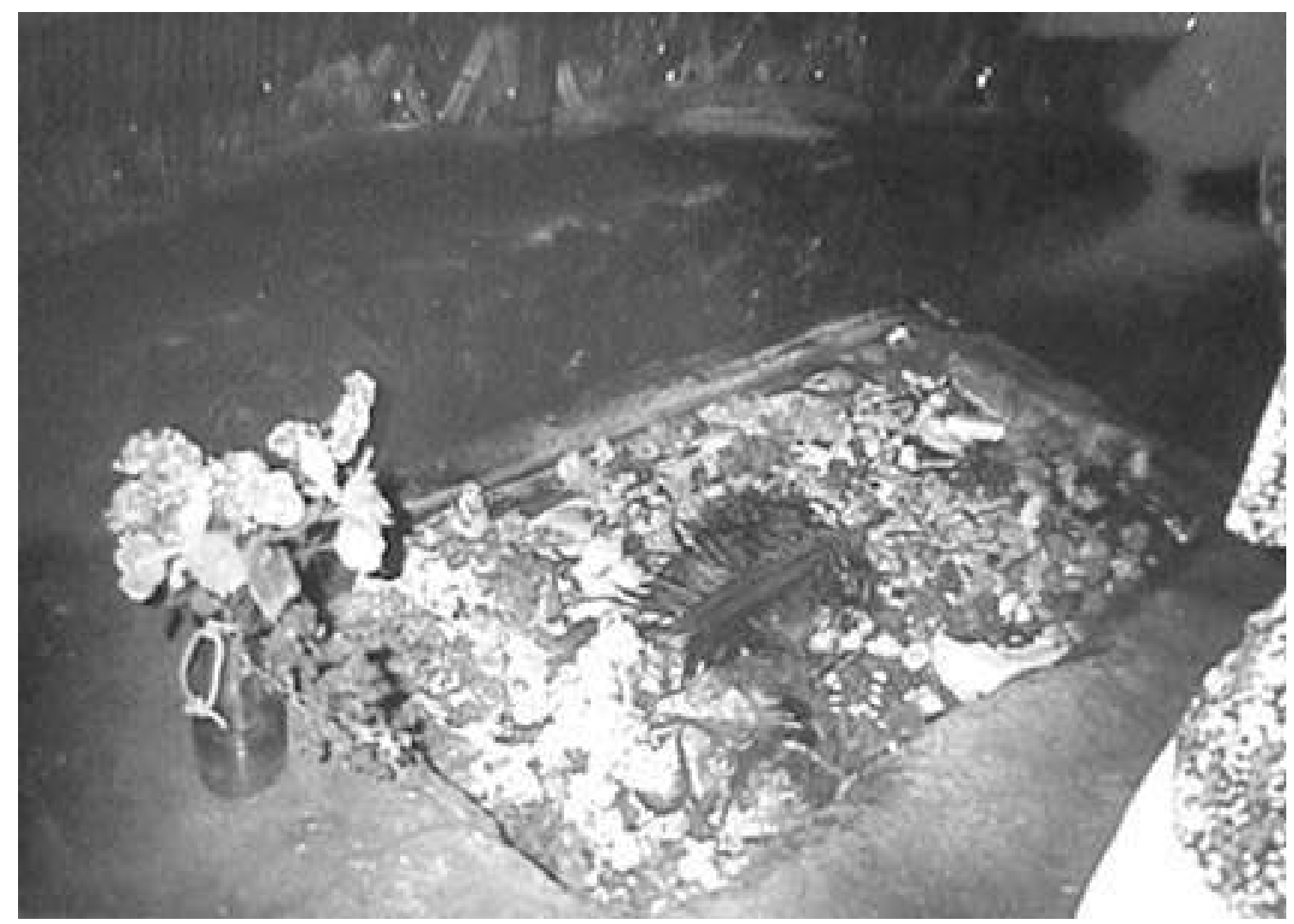

FIG. 6. - Tombe de Jimmy Stevens dans sa case. (C) M.K.Tabani. 
seul siège remporté par le Nagriamel en 1983 par Harry Karaeu, fut toutefois perdu en 1987. Karaeu, fils d'un chef coutumier de Tasmalum, se présenta en 1987 sous l'étiquette UPM (Union des Partis Modérés), prônant une fusion du Nagriamel avec ce parti et l'abandon de la spécificité de ses enjeux kastom locaux. Cette stratégie provoqua une scission au sein du Nagriamel, qui réussit à Karaeu puisqu'il fut élu contre Franky Stevens, fils cadet du patriarche de Vanafo.

La libération de Stevens offrit un sursaut au Nagriamel, qui vit élire Franky Stevens au parlement en 1991. Cette victoire fut obtenue grâce au dernier coup politique joué par Jimmy Stevens: la conclusion d'une alliance entre le Nagriamel et le tout nouveau Melanesian Progressive Party (MPP) de Barak Sope, ancien bras droit de Walter Lini qui avait créé son propre parti après ses dissensions avec le Vanuaaku Pati ${ }^{16}$. Les deux hommes élaborèrent cet accord en 1989, alors que Sope avait été condamné à une peine de prison et retrouva Stevens derrière les barreaux. Le succès électoral de cette nouvelle alliance du Nagriamel démontra que l'influence et la capacité de persuasion de Stevens sur les man-bush demeurait intacte ${ }^{17}$.

Franky Stevens fut élu en 1991, puis réélu en 1995. Entre temps, à peine Stevens père était-il décédé que de nouveau, de sérieuses dissensions se présentèrent au sein du Nagriamel à Santo. Franky fut accusé par les leaders traditionalistes du mouvement d'être un man-skul (chrétien) bien ignorant en matière coutumière, et donc illégitime quant à son autorité. Un schisme se produisit et Nakato Stevens, frère cadet de Franky, s'inspirant de la conduite de son père, prit la tête d'une liste dissidente au nom de la vraie kastom de Santo, lorsque Franky décida de s'allier au gouvernement. Ce groupe, tout en créant des dissensions, n'aboutit cependant pas à l'éclatement de la communauté de Vanafo. Son échec électoral fut patent, même si la légitimité de Franky en sortit affaiblie ${ }^{18}$.

La déliquescence actuelle du Nagriamel, accélérée par des différends pseudo-coutumiers et ses développements purement folkloriques, confirment une fois encore que l'histoire politique du Nagriamel a été largement déterminée par l'histoire personnelle de Jimmy Stevens, par l'ascension et la chute de ce personnage qui cumula toutes les contradictions et assumera tous les paradoxes, dans les sphères du privé comme du public, de l'individuel comme du collectif, du traditionnel et du moderne. Car Jimmy Stevens est l'individu à travers qui s'opère, pour ses sympathisants man-bush, la rupture des derniers

16. Rejeté par l'UPM, Stevens signa cette alliance avec Barak Sope qui lui offrit en retour des soins hospitaliers à Nouméa ( $c f$. Stevens, $1995: 241-242$ ). Sa justification fut imprégnée de ce perpétuel mélange de raisons coutumières et d'opportunisme politique qui fit également fortune à l'échelle nationale, et dont Stevens avait tracé la voie. Sur les injonctions de Stevens, Sope devait être considéré par les membres Nagriamel à la fois comme un frère dissident et un allié coutumier, au travers d'un obscur jeu de parenté (ibid : 241).

17. Van Trease précise la teneur de cet étonnant coup politique : «Le Nagriamel lui-même ne publia pas de programme séparé et utilisa celui du MPP, demandant seulement à ses supporters de se rallier derrière leur leader retrouvé, Jimmy Stevens, en soutenant les candidats Nagriamel. Stevens et ses associés avaient à peine eu le temps de consulter les partisans Nagriamel en dehors de Santo, provoquant une confusion bien compréhensible en regard de l'histoire passée. Les membres du mouvement Nagriamel étaient donc priés de s'allier avec quelqu'un qui avait été l'un des individus les plus puissants du VP lors de l'indépendance et avait beaucoup contribué à maîtriser la révolte, emprisonnant nombre de leurs leaders, y compris Jimmy Stevens » (Van Trease, $1995:$ 139).

18. Franky revendique à la fois la légitimité d'un politicien moderne, d'un successeur de son père à la tête du Nagriamel et d'un chef coutumier de Vanafo. Le village a d'ailleurs été hissé au rang de symbole. Il est devenu une sorte de village de « la belle au bois dormant». S'il demeure assez peuplé et si ses habitants apparaissent actifs, une partie du site a toutefois été spectaculairement muséifié, sa nouvelle finalité étant celle d'une attraction touristique. Depuis Luganville, on se rend à Vanafo en taxi ou en mini-van entre deux plongées sur l'épave de l'USS Coolidge (un ancien paquebot de croisière reconverti en transport de troupes pendant la guerre et qui sombra à l'entrée du port de Luganvillle). Franky a pour habitude d'accueillir chaleureusement les touristes qui, tous les jours, peuvent assister à une levée des drapeaux du Nagriamel dans une enceinte plantée de namele et nangaria, entretenue par des femmes habillées de jupes de feuilles, bien que le reste de la population soit vêtu à l'occidentale. A l'arrivée des touristes, Franky et l'ancienne garde rapprochée de Jimmy Stevens s'empressent de revêtir leur écusson Nagriamel, puis guident les visiteurs vers les ruines volontairement laissées en l'état de l'ancien Office Board du Nagriamel, là où fut installée Radio Vemarana saccagée ensuite par les troupes papoues. Le clou de la visite à Vanafo est constitué par la tombe de Jimmy Stevens, creusée dans sa case, où le corps resté à l'air libre après avoir subi un processus de dessiccation, repose sous un parterre de fleurs. Dans la case sont exposés les effets personnels de Stevens : ses costumes modernes sont impeccablement suspendus aux cloisons, ceints d'écharpes aux couleurs de la Nagriamel-Confederation indiquant la position de Chief-Minister de Stevens. Un portrait icônisé de Stevens, lui donnant l'allure d'un saint chrétien, est accroché au poteau central ; tout autour sont disposées les mâchoires de cochons aux incisives recourbées en arc de cercle, symboles du " grade coutumier» de Stevens. Enfin, à l'entrée, un livre d'or est présenté aux visiteurs où l'on retrouve les signatures de tout ce que le monde politique des états du Pacifique Sud compte de dignes représentants. La tombe de Jimmy Stevens à Vanafo est devenue un lieu de pèlerinage de première importance, une Mecque du traditionalisme à la mélanésienne. Ayant en partie hérité du goût de son père pour les symboles spectaculaires, Franky me confia avoir envisagé de consulter des spécialistes égyptiens pour embaumer efficacement le corps de son défunt père qui, dans ces conditions, pourrait alors être inhumé en haut du mont Tabwemassana à la suite d'une très grande cérémonie coutumière qui rappellerait au monde entier la grandeur de son père, promu au rang de Pharaon de la coutume. 
liens avec le passé pré-colonial. La poussée de fièvre qu'occasionna l'aventure du Nagriamel dans «l'histoire froide» des populations reculées de Santo, leur permit, par le biais des entreprises de Stevens, d'intégrer définitivement l'histoire universelle, celle du règne de l'individualisme et de l'exploitation des masses. L'apport idéologique fondamentalement nouveau de Stevens pour les man-bush comme pour l'ensemble de ses compatriotes, ressort parfaitement des conclusions de Beasant sur la décolonisation de ce confetti d'empire qui prit le nom de Vanuatu :

« La genèse de la rébellion, son règne court mais agité, et ses prolongements, décrivent dans leur essence la manière dont historiquement de simples individus peuvent interférer dans la politique du monde (world politics) » (Beasant, 1984: 151) [traduction de l'auteur].

Il nous reste à examiner comment à travers son cheminement personnel, Jimmy Stevens fut le premier ni-Vanuatu à user du procédé de l'indigénisation du legs traditionaliste colonial, technique qui par la suite fut largement reprise à tous les échelons de la vie sociale et politique à Vanuatu.

\section{J.T.P.S. Moses : Moli ou les dérives kastom du Nagriamel}

L'innovation que représentait l'idéologie du Nagriamel se laisse saisir à travers le cheminement personnel de Stevens et la spécificité de son pouvoir. À la lumière des nombreux documents et commentaires le concernant, on ne peut parler qu'en termes de carrière politique, pour évoquer son parcours. Il se voyait expressément investi d'un destin, d'une mission. Jimmy Stevens parlait en effet beaucoup et souvent, il se plaçait toujours au centre de ses thèmes favoris : que ce soit par rapport à sa position de «chef coutumier », son identification à son " peuple coutumier » ou son action pour un « renouveau coutumier " à travers la création du Nagriamel. Bref, si Jimmy Stevens ne s'est peut-être jamais exclamé « la coutume c'est moi », l'idée que s'en faisaient ses soutiens extérieurs était pourtant bien celle-là. Elle lui valut de la part des colons de Santo, le titre de " Prince des broussards », et plus abstraitement encore, celui de « Prince de la coutume » de celle des représentants français de l'administration condominiale ( $c f$. Hours, 1976 : 227). Ces mêmes affabulations reprises, et à peine déformées, dans la presse, au moment de la rébellion de Santo, firent de Jimmy Stevens « the leader of a stone-age army » (London 'Observer',
2 juin 1980). C'est en m'appuyant principalement sur les commentaires de Beasant, Van Trease et des ethnologues Kolig, Guiart, Hours et Bernard, et les allocutions écrites que nous a laissé Stevens, que je livrerai ma propre interprétation de la succession d'inventions que représentent le type inédit de leadership de ce dernier, la coutume du Nagriamel et l'instrumentalisation traditionaliste, sans précédent, d'une idéologie-kastom à des fins politiques.

À propos de Stevens et du Nagriamel, reviennent fréquemment des repères analytiques, comme " prophète », " cargo-cult», " mouvement indigène », et de façon plus systématique, celui de «big-man ». On retrouve également une série de termes à propos de ce qu'ils ne sont pas : un homme politique, un parti politique, une église, une nation. Commençons d'emblée par réfuter la notion de cargo-cult au regard des mouvements syncrétiques qui ont précédé le Nagriamel ( $c f$. Tabani, 2000 et 2001). Cette réfutation fut d'ailleurs exprimée par Stevens luimême :

«[Un jour,] il parla avec dédain des John Frum - avec lesquels il n'avait pas l'intention de s'associer - et des adeptes des cargo-cults en général : 'je n'attends pas de paquebot [steamer]. Je dispose déjà d'un paquebot noir. La terre est mon paquebot (Stevens, in Lindstrom, 1993 : 163).

Si Stevens se présente comme l'interprète des frustrations et des espoirs de ses sympathisants, ses alliances extérieures l'amèneront à dépasser le combat pour les terres pour lequel il fut initialement mandaté :

« Leur but correspondait à la signification que les ruraux donnent à la terre - l'accès aux biens matériels et un standard de vie plus élevé à travers sa mise en valeur » (Stevens, in Van Trease, 1987 : 155).

Le but de Stevens est de faire profiter les Mélanésiens des avantages de la société coloniale et de les émanciper au moyen d'une conscience noire :

«Le Nagriamel a existé avant moi. Mais il faut le réformer de nouveau - lui donner sa cohérence, son drapeau dont nous hissons les couleurs. Voilà mon travail. [...] Le Nagriamel sera pour vous mes enfants noirs » (ibid : 160).

Parmi les études les plus approfondies sur le Nagriamel, dont les autres commentateurs se sont largement inspirés, on retrouve en bonne place l'étude de Hours. Partant d'un récit autobiographique de quelques étapes de la vie de Jimmy Stevens, Hours trace un portrait psychosociologique de ce dernier. Il rapporte trois dimensions de la vie de Stevens à travers les trois 
ensembles patronymiques sous lesquels le personnage fut connu. Les deux premiers découpages paraissent judicieux, le troisième semble plus tendancieux, j'y viendrai en dernier.

Hours retrace la vie de Stevens en insistant sur la dimension schizophrénique apparente du personnage, qu'il attribue fondamentalement à sa condition initiale de métis dans un univers colonial où lui fut refusée l'éducation de la culture " supérieure », celle du groupe dominant. Jimmy Stevens est le nom qui se rapporte à la première partie de sa vie. Il est issu d'une grand-mère tongienne et d'un grand-père écossais, un marin qui ne reconnut pas sa descendance. Sa mère est la fille d'un chef des îles Banks, Stevens naquit à Santo dans le village de Rongofuro à Tasmalum, puis sa famille émigra à travers tout le nord de l'archipel, où son père exerça pour les Anglais tous les métiers subalternes des plantations. La famille Stevens se fixa dans les îles Banks avant que Jimmy ne la quitte, profitant de l'arrivée des troupes américaines qui le recrutèrent.

Jimmy le «boy», métis de métis et nonassimilé, réussit à s'affranchir du carcan strict de l'autorité familiale et de celui plus strict encore de l'ordre social de la société coloniale. Jimmy accéda, par ce biais, à des connaissances technologiques et put exercer des activités qualifiées. Sa jouissance d'une identité sociale reconnue sous le surnom d'American Jimmy, lui permit d'intégrer la condition de métis. Il y gagna, au contact des soldats noirs américains, une conscience de la négritude qui, par la suite, influença largement ses interprétations de la culture des man-bush.

La fin de la guerre le replongea néanmoins dans l'univers de ségrégation de la société blanche et métisse de Luganville. Il y connut les lourdes répercussions d'une vie urbaine et moderne. Car en étant au service des Blancs, il fut chargé de participer à l'exploitation des ressources foncières des communautés indigènes. À la croisée de deux mondes, son expérience professionnelle tourna à l'échec, suite à un grave accident de travail. Stevens prit conscience du «bidonville culturel» (Hours, 1976 : 219) dans lequel il se trouvait, tout en admirant l'unité que conservaient les communautés de l'intérieur de par leur relatif isolement. Hours conclut à propos de la fin de la jeunesse de Stevens, avant donc l'entreprise du Nagriamel et les désillusions qui l'accompagnèrent :

« Dépourvu d'une identité culturelle solide, victime plus que tout autre de la situation condominiale, tel se présente Jimmy Stevens dans la société locale. Habitué à vivre dès son plus jeune âge dans l'insécurité et sous la dépendance abstraite des deux pouvoirs omniprésents, il a retenu quelque chose de chacun des milieux où il est passé et de chacune de ses composantes de son état de métis » (ibid : 219).

Stevens en découvrant la coutume allait inventer une manière de tourner la loi à son profit. Il abandonna sa situation de "protégé britannique » en s'alliant au camp des métis francophones. La constitution et le développement du Nagriamel fut sa préoccupation la plus essentielle de toute la deuxième partie de son existence, lorsqu'en tant que Chief-President de la Féderation du Nagriamel il devint J.T.P.S. Moses, nom dont il signait tous les documents officiels. Avant d'apparaître pleinement aux yeux de ses sympathisants comme un homme providentiel, Stevens démarra sa carrière politique avec le statut d'une sorte d'observateurparticipant de la coutume de Santo. Mais il sut rapidement mesurer le bénéfice, en terme de prestige, qu'il pouvait tirer d'une revendication kastom et de son exploitation politique. Tubo, le nom de son père, lui offrit un repère identitaire local, par le biais des relations entretenues par son père avec le prophète Rongofuro qui avait sévi comme leader cultuel, à Santo, dans les années trente. Ce même patronyme lui permit également de revendiquer son ascendance tongienne $($ Tubo $=$ tupou) et les quartiers de noblesse et le sang royal hérités de sa grandmère. Pantuntun est le nom de sa mère et lui fournit son ancrage mélanésien, néo-hébridais et coutumier, son grand-père maternel ayant été un dignitaire des îles Banks ${ }^{19}$. Enfin, Moses est le nom qu'il prit lors de sa conversion à l'église Church of Christ, la première à avoir été entièrement dirigée par des Mélanésiens. C'est sur la multiplicité des référents identitaires qui composaient cette synthèse patronymique que Jimmy Stevens se forgea une identité politique de leader moderne prétendant à une légitimité coutumière.

\section{Jimmy Stevens, chef charismatique}

De toutes les dimensions du personnage de Stevens, la plus problématique et le plus ambiguë, fut celle emblématisée sous le nom de Moli. Ce nom correspond au titre le plus élevé dans le système de la hiérarchie des grades, l'institution rituelle majeure de cette partie de l'archipel. Il lui

19. Vienne précise que Jimmy Stevens, par sa mère est originaire du village de Veverau sur l'île de Mota. Le nom Pantuntun y est un nom personnel ancestral, récurrent dans la lignée à laquelle il se rattache ( $c f$. les généalogies données par Codrington [1891] et Vienne [1972]), et auquel s'attache un certain prestige (communication personnelle). 
fut donné enfant, au contact de la famille de Rongofuro, en hommage au fils décédé de ce dernier qui le portait. Stevens s'était redécouvert Moli lorsqu'il prit la place de Buluk en tant que chef du Nagriamel. Il entreprit alors de rendre à ce surnom son véritable référent traditionnel « en instituant des tueries cérémonielles de cochons à un rythme annuel » (Beasant, 1984 : 19), inspirés des cérémonies traditionnelles de prise de grades 20 .

Les commentaires de Hours ne sont pas clairs sur ce point. Ils présentent Stevens comme détenant un leadership qui demeure captif d'une dimension traditionnelle que lui-même a contribué à inventer. Car, par ailleurs, Hours dénonce les expressions spectaculaires de cette revendication coutumière, ces « cérémonies d'abattages de cochons effectuées devant des délégués condominiaux ou autres leaders politiques de hauts niveaux ne sont pas coutumières, elles sont des bouffonneries et des mises en scènes " (Hours, 1979 : 19), lorsqu'elles ne tournent pas carrément à la violence et au « terrorisme coutumier » (ibid: 18).

Hours tire de son postulat sur la traditionnalité du pouvoir de Stevens des prolongements théoriques sur la sécularisation des cultes millénaristes, comparables à ceux qui avait été avancés par Worsley (1977) et Guiart (1951). Ainsi, l'action de Stevens s'inscrirait dans un processus de transformation des cultes vers des formes d'organisation politique modernes, que le leader du Nagriamel, n'aurait pas su mener à son terme. Le Nagriamel serait demeuré un cargocult, et Stevens n'aurait pas atteint la dimension d'un homme politique capable d'intégrer une vision nationaliste de l'avenir des NouvellesHébrides. Hours présente également, dans son interprétation, un certain nombre d'éléments théoriques relevant de la typologie weberienne des ordres légitimes, qui lui font dire que Stevens, de par les dissensions qu'il engendra, n'atteignit jamais la dimension révolutionnaire ou nationaliste du leader charismatique. Qu'il se cantonna dans un rôle traditionnel de big-man mélanésien tout en empruntant, du fait d'influences extérieures, une voie réactionnaire.

Repris et modifié par Kolig, cet argument conduit sous la plume de ce dernier à présenter Jimmy Stevens comme un véritable leader charismatique (notion à laquelle il semble accorder la connotation de non-traditionnel), qui conserverait néanmoins la spécificité mélanésienne du big-man du modèle de Sahlins (achieved vs ascribed) (cf. Kolig, 1987 : 193-196). On mentionnera également sur ce point l'interprétation de Guiart, car ce dernier exprime une position bien plus radicale que celle des deux précédents commentateurs. Il affirme que l'on ne peut, en aucun cas, qualifier de charismatique le pouvoir de Stevens, ni lui attribuer le moindre ancrage traditionnel. Il considère que l'ascension de J.T.P.S Moses au rang de Moli relève de la pure charlatanerie, du mensonge et de l'opportunisme ( $c f$. Guiart, 1988 ; 1983) ${ }^{21}$.

Ce que l'on peut reprocher à l'analyse de

20. Les tueries pseudo-rituelles de cochons sont une pratique dont Stevens fut le premier à détourner la portée. Ils sont devenus dans la vie publique de Vanuatu le préalable incontournable de toutes manifestations officielles impliquant des enjeux kastom. Il semblerait que les prises de grades de Stevens correspondaient avec les festivités annuelles de célébration du Nagriamel, et par extension de la prise de pouvoir de son fondateur. La fréquence des abattages cérémoniels de cochons qu'il organisait, était fonction de visites officielles, d'inaugurations de diverses infrastructures modernes, de la conclusion d'alliances politiques, etc. Mentionnons la remarque de Derrida sur l'expression « small bisnis blong pig » dont se sert Stevens pour qualifier les sacrifices de cochons dans ces occasions secondaires, sachant que tous types de «bisnis blong pig » sont labellisés kastom : « L'expression 'bisnis blong pig' désigne habituellement la cérémonie traditionnelle de prise de grade, ou la hiérarchie entière : 'les Aobans appellent 'business belong pigs' (c'est-à-dire 'les affaires de cochons') le système des grades' (Bonnemaison, 1972 : 95) ; 'A l'origine de la carrière d'un big-man prestigieux, on trouve dans bien des cas la conversion d'un guerrier ou d'un sorcier mémorable au bisnes blong pik, selon une expression qui fait allusion au caractère de compétition économique que prend la hiérarchie des grades' (Vienne, 1984: 308). Ici, [en une occasion officielle secondaire], le smol bisnis blong pig ne désigne apparemment que l'abattage des porcs, sans que cela ait un rapport direct avec le rituel traditionnel » (Derrida, 1990 : 172).

21. L’interprétation proposée par Guiart sur le Nagriamel et sur les événements de Santo en 1980 mérite quelques commentaires. Certains de ses points de vue ne manquent pas d'intérêt, lorsqu'il écrit, par exemple, que : "Jimmy Stevens monta une remarquable opération de relations publiques pour se faire reconnaître Moli, c'est à dire dignitaire dans la hiérarchie des grades, ce que les Européens traduisent par le mot chef, avec tout ce que cela comporte pour eux d'autorité incontestée » (Guiart, 1983 : 175). « En réalité [Jimmy Stevens] connaissait mal la vieille société mélanésienne de Santo. Cependant, il était à même d'imaginer quelques principes, plus ou moins valides selon les îles, qu'il exposait publiquement et qu'il illustrait comme paraboles de ce que devait être la kastom, construisant ainsi à partir de ce concept une sorte d'image de pure science-fiction sociale [...]. Le Nagriamel n'était ni une coutume ni une tradition, mais en lui faisant jouer quotidiennement un rôle de fabrication de croyance, on le rendait politiquement dangereux. » (Guiart, $1988: 201$ ).

Mais il convient surtout de souligner que Guiart ne donne aucune intention théorique à son propos qui se compose surtout d'invectives. Il accuse Stevens d'escroquerie à grande échelle et même d'assassinat, ou lorsqu'il assimile le Vemarana à une association de malfaiteurs : "[La] soi-disant police [mise en place par l'état-major du Vemarana 'composé d'incapables patentés'(Guiart)] servit soit à violer en public deux jeunes femmes auxiliaires du Vanuaaku Pati, soit à brutaliser les policiers officiels faits prisonniers au saut du lit, soit à jouer avec et casser des voitures officielles ou celles des sympathisants du parti 
Hours, est qu'elle s'attache à la description d'un contexte moderne et de ses enjeux idéologiques, en introduisant des concepts ethnologiques se rapportant à un contexte spécifiquement traditionnel. Au travers d'une telle traditionalisation du pouvoir de Jimmy Stevens, ce qui est perdu de vue est le charisme idéologisé de l'homme providentiel moderne, du président-plébiscitaire de Weber, qui est une conséquence et non une cause de la rationalité de son organisation ( $c f$. Weber, $1995: 350$ ).

Ce qui doit retenir l'attention est cette capacité de mobilisation dans le Nagriamel qui permet à Hours de ramener ce mouvement au rang d'un néo-cargo-cult. Le développement de son administration et de ses appuis extérieurs crût à mesure que Stevens réinventa la kastom et renforçait son pouvoir personnel. C'est cette combinaison de revendications à la fois philocoutumières et autocratiques qui constituent, véritablement, l'instrumentalisation traditionaliste des traditions. Elles valent à Stevens la qualité de précurseur idéologique à un niveau régional des pratiques culturelles généralisées et codifiées, aujourd'hui, à l'échelle nationale de Vanuatu (cf. Tabani, $2000 ; 2001)$.

\section{Jimmy Stevens et la politique}

Jimmy Stevens cherchait à faire de la coutume sa propre loi. Pour affirmer la légalité du Nagriamel, il recourait fréquemment à des constructions sociales et politiques totalement imaginaires :

«Le Nagriamel fait partie d'une fédération, d'un État que nous appelons Nakatoro. Dans la constitution du Nagriamel, Natakaro représente toutes les Nouvelles-Hébrides. Hélas, quand les Blancs sont arrivés ici, ils ont remplacé le nom et la notion de Natakaro par 'Nouvelles-Hébrides'. Avant que les Blancs n'arrivent, il avait une loi que certains nommaient Natamata ou Namangi qui excluait toute autre loi pour régir le pays »(Stevens, in Bernard, 1983 : 73).
Stevens, tout en posant des revendications d'inspiration démocratiques (que l'on retrouvait par exemple dans des slogans du type «individual rights for everybody » lancés en des occasions publiques par les membres du Nagriamel) (cf. Beasant, $1984: 61$ ), cherchait à tout prix à conserver l'aspect communautaire du Nagriamel, en plaçant la coutume au-dessus de toute autre considération : "Si vous voyez quelqu'un aller nu, cet homme est un des vôtres, vous peuple nu de Santo. Quelqu'un qui va nu, vous pouvez voter pour lui » (Stevens, in Philibert, 1990 : 458). Il s'agissait pour Stevens de préserver les man-bush de la " politique » (politik), en assumant seulement lui-même cette "contagion ». Cette position fut un facteur déterminant de l'orientation réactionnaire prise par le Nagriamel, et la stratégie ultime de Stevens pour instaurer une sorte de dictature de la coutume :

« Je remarquais [en considération de l'évolution politique des Nouvelles-Hébrides avant l'indépendance] que de nombreux partis politiques émergeaient, chacun avec son point de vue différent. Je m'inquiétais de ce que cela risquait d'affecter l'étatmajor du Nagriamel à Vanafo et suggérait en conséquence d'établir un groupe séparé à Luganville. [...] L'objectif de ce bureau [du Vemarana] était de s'occuper des questions politiques et de s'assurer que ces questions n'atteignent pas le reste du mouvement. J'étais le leader du Nagriamel et en même temps Président du Vemarana » (Stevens, 1995 : 237).

Appliquée à des situations concrètes, la position de Stevens d'une individualité incarnant organiquement son peuple, lui permettait de justifier de ses actes les plus arbitraires, comme par exemple l'expulsion de Mélanésiens originaires des autres îles :

«Parce que c'est la coutume. Natakaro a parlé ! Natakaro dit: 'vous pouvez rester ici si vous vous conformez à la coutume, mais si vous voulez installer votre pouvoir contre la coutume, vous devez vite retourner chez vous'. Ceci n'est pas la déclaration d'un seul homme mais la voix de la coutume, de la coutume

majoritaire, à déféquer dans les lieux de culte protestant, à pratiquer le cambriolage avec effraction dans les maisons privées ou les docks des maisons de commerce; à organiser de grandes soûleries avec le l'alcool ou le vin volés, tout en mettant à la broche le bétail abattu appartenant aux ennemis supposés du Vemarana. Il n'y a pas eu une tentative cohérente de gestion, sinon, pour user de la menace et de la violence pour faire payer à tous les habitants de la région l'impôt du Vemarana » (Guiart, 1983 : 203). Enfin, il faut dire que l'ensemble des affirmations de Guiart se voient quelque peu discréditer lorsqu'il encense la répression des troupes papoues à la manière d'une « participation mythique » pour reprendre l'expression de Leenhardt, son vieux professeur : « Les Papous avaient fait mieux que les Blancs. Du point de vue du gouvernement du Vanuatu et de l'affermissement de l'unité nationale, le règlement du problème né de la sécession par le recours à une armée noire, contre les Européens pour l'essentiel, [...] correspondait à la vieille prophétie John Frum des soldats noirs sortant de la montagne ou du volcan pour chasser les Blancs du Pays » (ibid: 208).

De telles fictions idéologico-ethnologiques se retrouvent cependant chez des ethnologues ayant soutenu chacun des deux camps. J'en donnerais pour seul exemple la traditionalisation que Bonnemaison fait subir dans son interprétation à la sécession de Santo : «Les groupes 'modérés', prenant appui sur le fait que la tradition mélanésienne repose sur la voie du consensus, refusèrent le verdict électoral. Dans la société traditionnelle en effet, aucune décision ne peut être prise sans que l'accord de tous n'ait été obtenu. La minorité si elle persiste dans son désaccord, n'a d'autre issue que de se taire ou de s'exclure. Dans le premier cas, elle admet implicitement la suprématie de la thèse inverse, dans le second, elle fait sécession » (Bonnemaison, 1986 : 454). 
qui a toujours existé... Aussi, aujourd'hui, les gens qui habitent Santo doivent voter pour la coutume de Santo. S'ils veulent voter 'politique', ils doivent retourner chez les gens du Vanuaaku. Moi je crois que ce parti vient de Nouvelle Zélande et d'Australie, mais voilà, les Noirs ne peuvent pas aller vivre en Nouvelle Zélande ou en Australie...» (Stevens, in Bernard, $1983: 73)$.

Les contradictions culturelles qui ont miné tout développement cohérent de ce mouvement, les incompatibilités de ses alliances, ne lui ont jamais permis d'atteindre du point de vue idéologique une quelconque dimension nationaliste, d'où le fiasco de la rébellion. Sur ce plan, le véritable tournant dans l'évolution politique du Nagriamel se situe vers 1976, lors de sa rupture définitive avec le Vanuaaku Pati et les élites occidentalisées émergentes. Le VP ne cessera depuis ce moment de dénoncer Jimmy Stevens pour avoir rejoint les Français, et réciproquement, ce dernier n'aura de cesse d'accuser les membres du VP d'avoir «quitté la coutume et rejoint l'homme blanc » (cf. Van Trease, $1987: 168)$. Ce n'est sans doute pas plus à cause de la coutume, comme le suggère Hours, que le Nagriamel n'est pas parvenu à « sublimer [les réalités vécues des man-bush] sous la forme d'un mythe romantique nationaliste », que du fait que « le Nagriamel est un groupement de broussards et ses préoccupations politiques visent d'abord à l'organisation politique des man-bush » (Hours, 1974 : 238).

L'incapacité de Stevens à former ou rallier une élite moderne mélanésienne dans le sillon du Nagriamel, ou encore à trouver des arrangements avec les églises qui le soupçonnaient d'encourager et de vouloir revenir au paganisme (cf. Beasant, $1984: 24$ ) 22, ne lui a permit d'entrevoir d'autres solutions que de concentrer entre ses seules mains un assemblage d'autorité moderne et de traditions inventées, en s'attribuant un pouvoir quasi-monarchique. Son rejet par les élites anglophones l'entraîna à multiplier les alliances extérieures, à se reposer sur des colons et des cadres étrangers, et à rechercher la bienveillance des autorités coloniales françaises. Tout comme le Vanuaaku Pati nationaliste, mais sur une base différente, le Nagriamel demeurait dans la dépendance des Blancs : " Je veux que mon peuple se défasse des règles condominiales, non pas une indépendance pour chasser l'homme blanc, mais pour que le peuple noir ne soit plus sous son contrôle » (Stevens, in Van Trease, $1987: 163$ ).

Les fortes sommes d'argent reçues par Stevens de ses soutiens extérieurs n'ont jamais servi à la mise en place de plans de développement fructueux ou à la construction d'infrastructures durables, mais seulement à développer la structure administrative du Nagriamel, à l'organiser en un groupe de pression coutumier pour soutenir le pouvoir autocratique de Stevens. Il est intéressant de relever que c'est sur l'aspect du contrôle des finances du Nagriamel par Stevens, que ce dernier se vit reconnaître sa véritable qualité d'homme politique, au travers des jalousies qu'il déclenchait chez ses pairs qui voyaient en lui « l'individu le plus corrompu que Vanuatu n'ait jamais connu, et tout le monde le sait » (in Van Trease, 1987: 165) ${ }^{23}$. Les vues les plus objectives formulées sur Jimmy Stevens sont sans doute celles de Van Trease, qui tout en dénonçant les menées séparatistes de Stevens et les manipulations qu'il subissait de la part de ses alliés extérieurs, reconnaît très honnêtement l'absence chez lui d'une volonté de flouer ses sympathisants. Il se serait laissé entraîner dans un jeu politicien sans bien en maîtriser les règles, ambitionnant simplement au départ un pouvoir personnel comme tant d'autres responsables politiques modernes. $\mathrm{Vu}$ son intérêt, je livre in-extenso un extrait du commentaire de Van Trease (1987: 165-167) sur ce point :

« Avant de laver Jimmy Stevens des accusations de 'politicien corrompu', il faut se demander si sa conduite peut être considérée comme plus malhonnête que celle de toute personne ayant la même position de

22. D'après Bernard Vienne (comm. pers.) : « Contrairement aux affirmations du Nagriamel — et de son leader — et aux allégations de ses supporters dans le monde des 'petits blancs' et métis de Santo, Jimmy Stevens et son mouvement n'ont jamais réussi à avoir une réelle emprise sur les populations anglicanes des îles du Nord et en particulier sur les gens des îles Banks ; pas même sur les communautés de man Bankis installées au Mango à Luganville ou travaillant dans les plantations européennes de la côte Est. Cet 'échec' est tout un problème en soi. Jimmy Stevens ne se faisait guère d'illusion sur une telle perspective. Il s'efforçait de 'tirer profit' de certaines dissensions au sein du Vanuaaku Pati pour mieux convaincre de son aura politique ses 'supporters potentiels' dans l'entourage de l'administration coloniale qui étaient tout prêts à entendre ce qu'ils avaient envie de croire. L'analyse des résultats électoraux - même si on a pu les contester - confirme bien l'étroitesse et le caractère local de l'emprise du Nagriamel et du rayonnement de son leader ».

23. Il ressort de la Synthèse Mensuelle de la Résidence de France de janvier 1975, que c'était encore une fois cet aspect du personnage de Stevens qui présentait le plus d'intérêt aux yeux des autorités coloniales françaises : « Dans l'esprit flegmatique des Mélanésiens, l'attitude de Stevens, ses coûteux voyages, ses maîtresses (qui sont fêtées dans le calendrier du mouvement comme 'femmes qui se sont sacrifiées à la cause') font douter de son intégrité et de la sincérité de son attachement aux intérêts de ses demi-frères de sang. En ce qui me concerne, je ne suis pas pressé de le voir disparaître de la scène, dans la mesure où il risque d'être remplacé par des leaders plus forts et plus sincères, qui ne pêcheront pas par avidité, esprit vénal et fourberie, caractéristiques qui font de lui une proie facile pour des investisseurs sans scrupules » (in Van Trease, 1987: 165). 
pouvoir et si le degré apparemment immoral de ces décisions est dû à l'ignorance et l'inexpérience, ou bien s'il a agi avec une volonté consciente de décevoir et de frauder. [...] Son niveau de vie n'était en aucun cas extravagant [...]. Les aides substantielles de l'étranger ne furent pas, au départ, dépensées pour le confort personnel du leader mais plutôt pour alimenter l'organisation et pour aider les membres du Nagriamel grâce à de petits projets de développement. En effet, de par ses nombreuses femmes et ses enfants, il aurait eu des dépenses bien supérieures à la moyenne et n'auraient pu être couvertes que par une aide financière du Nagriamel — son âge, sa santé fragile, ses obligations en tant que Président de l'organisation ne lui permettaient pas de produire sa propre nourriture ou de tirer de l'argent de ses récoltes. Dans tous les cas, il n'est pas surprenant que ses partisans, dont le nombre dépassait les 20000 personnes, voulurent aider leur leader et honorer son statut en lui procurant de quoi vivre normalement. Il est en effet courant que les leaders politiques, une fois dans une position de pouvoir, aient du mal à vivre selon leurs idéaux » [traduction de l'auteur].

Sur un plan idéologique, on retrouve dans ce mouvement les accents culturels d'une revendication de la négritude qui, si l'entreprise du Nagriamel avait réussi, aurait pu fournir les bases au développement d'une idéologie ethniciste. Dans l'univers du colonialisme français, Jimmy Stevens pourrait être situé entre le Bachaga Bouelem et Léopold Senghor, et non pas comparé à Castro comme le suggère Kolig (1987) (sauf peut-être pour sa barbe). Stevens se voulait un roi des îles, noir et progressiste, gage d'un patronage colonial à visage humain. On doit lui reconnaître une certaine pondération, voire une certaine tolérance, en comparaison de l'attitude des élites nationalistes de Vanuatu. Il éleva ses conceptions traditionalistes au rang d'une utopie personnelle et non d'une idéologie d'État.

Laissons les derniers mots au patriarche de Vanafo qui, à la grande époque de son mouvement, était encensé sur les ondes de Radio Vemarana, où le disc-jockey allemand Bob Schnell, alias «Lord Paw-Paw », veillait scrupuleusement à jouer tous les soirs en fin de programmes, en hommage à Stevens, le tube disco « Daddy Kool » du groupe Boney M (cf. Beasant, 1984 : 97) :

«En ce qui concerne le Nagriamel, je crois que ce mouvement continuera d'aller de l'avant. Cependant, pour m'assurer que l'on continue dans la voie tracée par Moli Stevens, j'ai tué cent cochons et dix autres pour rendre royal le nom de Stevens. En bislama, natamata signifie royal. Après ma mort, mes descendants natamata seront ainsi traités pour toutes les générations à venir. [...]. Ce plan est étroitement lié à ma famille [...]. Avant mon arrestation, j'avais $25 \mathrm{fem}$ mes, mais la Force Mobile m'a fait beaucoup d'histoires à leur sujet et je décidais donc de prendre un nouveau groupe de femmes. J'ai de nombreux enfants, dont 15 garçons. Je suis très attaché au nombre 15 . Je suis né le 15 juin. C'est le 15 janvier que les chefs de Vanafo placèrent leurs mains sur moi. Pour les Blancs, un ensemble est dix. Pour les Israéliens c'est 12. Pour moi c'est 15 . J'organise mon travail et ma vie par étape de quinze. Et bien sûr, il y a quinze îles Nagriamel. Je souhaite un jour établir dans chaque district un de mes fils avec sa famille » (Stevens, 1995 : 243).

\section{Conclusion}

Le cas de Jimmy Stevens est exemplaire à plus d'un titre. Il témoigne de la sécularisation du charisme, c'est-à-dire de sa rationalisation pour en extirper les éléments étrangers à l'économie, et de l'accompagnement du développement bureaucratique par une idéologie traditionaliste. La dimension individuelle du pouvoir de Stevens ne correspondait plus au fond de prestige et au pouvoir personnel du big-man, mais à une personnification idéologique à son mouvement, le Nagriamel. Jimmy Stevens fut beaucoup plus proche de ces Blancs « Rois des îles » que connurent ces archipels au siècle dernier, que du bigman charismatique de la tradition ethnologique formalisée par Sahlins (1962). Le symbolisme inventé par Stevens pour son action semblait davantage obéir au modèle d'une féodalité traditionaliste sous tutelle coloniale.

À l'égard de l'invention des traditions relatives au pouvoir, de la naturalisation des rapports modernes de domination, le Nagriamel et l'action de son inspirateur, représentent le stéréotype colonial d'une idéologie traditionaliste bien rodée, qui a été reprise et adaptée au contexte post-colonial. Les prolégomènes de ses constructions ont été depuis lors folklorisés, muséifiés et font désormais partie des curiosités touristiques offertes par le nouvel État. Le mouvement de « libération » du peuple du dark bush, la « résistance» des men blong darkness, sont aujourd'hui livrés à l'appréciation des visiteurs de la tombe de Jimmy Stevens dans la casemausolée d'un Colombey des tropiques. Lorsqu'il fut enterré en 1994, l'homélie qui lui fut rendue par la presse officielle le présenta comme le 'Roi du mouvement Nagriamel', comme 'l'aîné de la famille royale de Vanafo' " (Vanuatu Weekly, 5 mai 1994). 


\section{BIBLIOGRAPHIE}

BABADZAN, Alain, 1988. «Kastom and NationBuilding in the South Pacific ». in Guidieri, R. et al (eds.) Ethnicities and Nations: Processes of InterEthnic Relations in Latin America, Southeast Asia, and the Pacific. Austin : University of Texas Press, pp.199-228.

BEASAnt, John, 1984. The Santo Rebellion, an Imperial Reckoning. Honolulu : Hawaii University Press.

BERnARD, Pascal, 1983. Le Nagriamel: tradition et nationalisme au Vanuatu. Thèse de troisième cycle soutenue à l'Université Paris-X-Nanterre.

Bonnemaison, Joël, 1972. «Systèmes des grades et différences régionales en Aoba». Cahiers ORSTOM, vol.XI, nº 1 , pp.87-108.

—, 1986. Les fondements d'une identité. Territoire, histoire, et société dans l'Archipel de Vanuatu (Mélanésie) : L'Arbre et la Pirogue, essai de géographie culturelle, tome I. Paris : ORSTOM.

Codrington, Robert Henry, 1891. The Melanesians : Studies in their Anthropology and Folklore. Oxford : Clarendon Press.

DERrIDA, Jean, 1990. Les étapes du processus de sécularisation au travers de l'analyse comparative de deux syncrétismes mélanésiens: Tanna, Santo; Vanuatu. Mémoire de Maîtrise, Université Paris-XNanterre.

GuiART, Jean, 1951. «Forerunners of Melanesian Nationalism », Oceania, vol.22, n², pp.81-90.

_, 1958. Espiritu Santo (Nouvelles-Hébrides). Paris : Plon.

_, 1983. La terre est le sang des morts, Nouvelle Calédonie du passé au présent. Paris : Anthropos.

—, 1988. « Réponse à l'article de E. Kolig ». Journal de la Société des Océanistes, vol.85, pp. 201-204.

Henninghan, Stephen, 1992. «Vanuatu. Political Review ». The Contemporary Pacific, vol.4, $\mathrm{n}^{\circ} 2$, pp.403-406.

Hours, Bernard, 1974. «Un mouvement politicoreligieux néo-hébridais : le Nagriamel ». Cahiers ORSTOM, série Sciences Humaines, vol.11, n³-4, pp. 227-242.

_, 1976. «Leadership et Cargo cult : l'irrésistible ascension de J.T.P.S. Moïse ». Journal de la Société des Océanistes, vol.32, n²2, pp.207-231.

—, 1979, «Custom and Politics in the New Hebrides ». Pacific Perspectives, $\mathrm{n}^{\circ}$ 8, pp. 15-20.

KeEsing, Roger M., 1989. «Creating the Past : Custom and Identity in the Contemporary Pacific». Contemporary Pacific, ${ }^{\circ} 1$, pp. 19-42.

Kolig, Erich, 1981. "The Paradox of Santo, Vanuatu ». Pacific Perspective, vol. 10, nº1, pp. 5761.

_, 1987. « Kastom, Cargo and the Construction of Utopia on Santo,Vanuatu : The Nagriamel Movement ». Journal de la Société des Océanistes, vol.85, pp.181-204.
Lindstrom, Lamont, 1993. Cargo Cult. Strange Stories of Desire from Melanesia and Beyond. Honolulu : University of Hawaii Press.

_, 1996. The American Occupation of the New Hebrides (Vanuatu). Christchurch (N.Z.) : University of Canterburry, Macmillan Brown Centre for Pacific Studies.

Miller, Graham J., 1948. « Naked Cult in Central West Santo ». Journal of Polynesian Society, vol.57, n4, pp. 330-341.

PAITEL, Patrick, 1985. L'enjeu Kanak. Paris : Éditions France-empire.

Philibert, Jean-Marc, 1990. « The Politics of Tradition: Toward a Generic Culture in Vanuatu ». in Manning, F. \& Philibert, J.-M. (eds.), Customs in Conflict: The Anthropology of a Changing World. Peterborough : Broadview Press, pp.251-273.

Plant, Chris, 1977. «The Nagriamel Federation: New Country Old Story ». Pacific Perpective, vol.6, $\mathrm{n}^{\circ} 1, \mathrm{pp} .49-57$.

RAFF, E., 1928. « Extract from a Letter from Rev. E. Raff, Vila New Hebrides, 10 janvier $1924 »$. in Williams, F.E., Orokaiva Magic. Oxford : Clarendon Press, pp. 100-101.

ROBERT, Jean-Jacques, nd., « Un paradis perdu ». [ms] $9 \mathrm{p}$.

SAHLINS, Marschall, 1962. «Poor Man, Rich Man, Big-Man, Chief : Political Types in Polynesia and Melanesia ». Comparative Studies in Society and History, vol.5, pp. 285-303.

STEVENS, Jimmy, 1995. « Le mouvement Nagriamel ». in Van Trease, H. (ed.), La politique mélanésienne : stael blong Vanuatu. Christchurch/Suva: Macmillan Brown Centre for Pacific Studies and Institute of Pacific Studies, University of South Pacific, pp. 237-244.

TABANI, Marc Kurt, 1999. « Kastom et traditionalisme: Quelles inventions pour quelles traditions à Tanna, Vanuatu ». in, Les politiques de la tradition : sur la construction des identités nationales et des identités culturelles dans le Pacifique, Journal de la Société des Océanistes 109, pp. 121-132.

_ 2000. «Walter Lini, la coutume de Vanuatu et le Socialisme mélanésien », Journal de la Société des Océanistes 111, pp. 173-194.

_, 2001. Les pouvoirs de la coutume : traditionalisme et identités culturelles à Vanuatu. Paris : L'Harmat$\tan$ (sous presse).

Van Trease, Howard N., 1987. The Politics of Land in Vanuatu : from Colony to Independance. Suva : Institute of Pacific Studies, University of the South Pacific.

—, 1995. « Les élections ». in Van Trease, H.N. (ed.), La politique mélanésienne: stael blong Vanuatu. Christchurch/Suva : Macmillan Brown Centre for Pacific Studies and Institute of Pacific Studies, University of South Pacific, pp. 127-168. 
VIENNE, Bernard, 1972. Les formes du pouvoir et de l'autorité dans les communautés paysannes des îles Banks (Nouvelles-Hébrides). Nouméa: Centre ORSTOM.

_, 1984, Gens de Motlav. Idéologie et pratique sociale en Mélanésie. Paris, ORSTOM/Société des Océanistes.

Weber, Max, 1995. Économie et société, trad. de l'all. sous la direction de Jacques Chavis et d'Éric de Dampierre [1971]. Paris : Plon.
White, Geoffrey M. et Lamont Lindstrom (eds.), 1997. Chiefs Today : Traditional Pacific Leadership and the Post-Colonial State. Stanford: University Press.

Worsley, Peter, 1977. Elle sonnera la trompette, le culte du Cargo en Mélanésie. Traduit de l'angl. par Brigitte Duval. Paris : Payot. 